\title{
THE LOWER BOUND FOR THE MODULUS OF THE DERIVATIVES AND JACOBIAN OF HARMONIC INJECTIVE MAPPINGS
}

\author{
MIODRAG MATELJEVIĆ
}

Research partially supported by MNTRS, Serbia, Grant No. 174032

This is a very rough version.

\begin{abstract}
We give the lower bound for the modulus of the radial derivatives and Jacobian of harmonic injective mappings from the unit ball onto convex domain in plane and space. As an application we show co-Lipschitz property of some classes of qch mappings. We also review related results in planar case using some novelty.
\end{abstract}

Throughout the paper we denote by $\Omega, G$ and $D$ open subset of $\mathbb{R}^{n}, n \geq 1$.

Let $B^{n}(x, r)=\left\{z \in \mathbb{R}^{n}:|z-x|<r\right\}, S^{n-1}(x, r)=\partial B^{n}(x, r)$ (abbreviated $S(x, r)$ ) and let $\mathbb{B}^{n}, \mathbb{S}=\mathbb{S}^{n-1}$ stand for the unit ball and the unit sphere in $\mathbb{R}^{n}$, respectively. In particular, by $\mathbb{D}$ we denote the unit disc $\mathbb{B}^{2}$ and $\mathbb{T}=\partial \mathbb{D}$ we denote the unit circle $\mathbb{S}^{1}$ in the complex plane.

For a domain $D$ in $\mathbb{R}^{n}$ with non-empty boundary, we define the distance function $d=d_{D}=\operatorname{dist}(D)$ by $d(x)=d(x ; \partial D)=\operatorname{dist}(D)(x)=\inf \{|x-y|: y \in \partial D\}$; and if $f$ maps $D$ onto $D^{\prime} \subset \mathbb{R}^{n}$, in some settings it is convenient to use short notation $d^{*}=d^{*}(x)=d_{f}(x)$ for $d\left(f(x) ; \partial D^{\prime}\right)$. It is clear that $d(x)=\operatorname{dist}\left(x, D^{c}\right)$, where $D^{c}$ is the complement of $D$ in $\mathbb{R}^{n}$. Let $G$ be an open set in $\mathbb{R}^{n}$. A mapping $f: G \rightarrow \mathbb{R}^{m}$ is differentiable at $x \in G$ if there is a linear mapping $f^{\prime}(x): \mathbb{R}^{n} \rightarrow \mathbb{R}^{n}$, called the derivative of $f$ at $x$, such that

$$
f(x+h)-f(x)=f^{\prime}(x) h+|h| \varepsilon(x, h)
$$

where $\varepsilon(x, h) \rightarrow 0$ as $h \rightarrow 0$. For a vector-valued function $f: G \rightarrow \mathbb{R}^{n}$, where $G \subset \mathbb{R}^{n}$, is a domain, we define

$$
\left|f^{\prime}(x)\right|=\max _{|h|=1}\left|f^{\prime}(x) h\right| \quad \text { and } \quad l\left(f^{\prime}(x)\right)=\min _{|h|=1}\left|f^{\prime}(x) h\right|,
$$

when $f$ is differentiable at $x \in G$. Occasionally we use the notation $\Lambda_{f}(x)$ and $\lambda_{f}(x)$ instead of $\left|f^{\prime}(x)\right|$ and $\ell\left(f^{\prime}(x)\right.$ (in particular in planar case) respectively.

For $x \in R^{n}$, we use notation $r=|x|$. We say that Jacobian $J$ of mapping on a domain $\Omega$ satisfies minimum principle if for every compact $F \subset \Omega$ we have $\inf _{F} J \geq \inf _{\partial F} J$. A $C^{1}$ (in particular diffemorphisam) mapping $f: \Omega \rightarrow \Omega^{*}$ is $K$-qc iff

$$
\left|f^{\prime}(x)\right|^{n} / K \leq|J(x, f)| \leq K \ell\left(\left(f^{\prime}(x)\right)^{n}\right.
$$


holds for every $x \in \Omega$. For $\xi \in \mathbb{S}$, define

$$
h_{b}(\xi)=h^{*}(\xi)=\lim _{r \rightarrow 1} h(r \xi)
$$

when this limit exists.

The directional derivative of a scalar function $f(\mathbf{x})=f\left(x_{1}, x_{2}, \ldots, x_{n}\right)$ along a vector $\mathbf{v}=\left(v_{1}, \ldots, v_{n}\right)$ is the function defined by the limit

$$
\nabla_{\mathbf{v}} f(\mathbf{x})=\lim _{h \rightarrow 0} \frac{f(\mathbf{x}+h \mathbf{v})-f(\mathbf{x})}{h} .
$$

If the function $f$ is differentiable at $x$, then the directional derivative exists along any vector $v$, and one has

$$
\nabla_{\mathbf{v}} f(\mathbf{x})=\nabla f(\mathbf{x}) \cdot \mathbf{v}
$$

where the $\nabla$ on the right denotes the gradient and - is the dot product. Intuitively, the directional derivative of $\mathrm{f}$ at a point $x$ represents the rate of change of $f$ with respect to time when it is moving at a speed and direction given by $v$. Instead of $\nabla_{\mathbf{v}} f$ we also write $D_{\mathbf{v}} f$. If $v=\frac{x}{|x|}, x \neq 0, g(t)=f(x+t v)$ and $D_{\mathbf{v}} f(x)$ exists, we define $\partial_{r} f(x)=D_{\mathbf{v}} f(x)=g^{\prime}(0)$.

Let $\Omega \in \mathbb{R}^{n}$ and $\mathbb{R}^{+}=[0, \infty)$ and $f, g: \Omega \rightarrow \mathbb{R}^{+}$. If there is a positive constant $c$ such that $f(x) \leq c g(x), x \in \Omega$, we write $f \preceq g$ on $\Omega$. If there is a positive constant $c$ such that

we write $f \approx g$ (or $f \asymp g$ ) on $\Omega$.

$$
\frac{1}{c} g(x) \leq f(x) \leq c g(x), \quad x \in \Omega
$$

O.Martio 24 observed that, every quasiconformal harmonic mapping of the unit disk onto itself is co-Lipschitz. Then the subject was intensively studied by the participants of Belgrade Analysis Seminar, see for example [27, 21, 31, 34] and the literature cited there. In particular Kalaj and Mateljević, shortly KMapproach, study lower bound of Jacobian. The corresponding results for harmonic maps between surfaces were obtaind previously by Jost and Jost-Karcher [14, 15]. We refer to this results shortly as JK- result (approach). Recently Iwaniec has communicated the proof of Rado-Kneser-Choquet theorem (shortly Theorem RKC), cf. [11, Iwaniec- Onninen cf. [12. We refer to this communication shortly as IwOnapproach. It seems that there is some overlap between KM- results with [11, 12] and 14, 15. (we will shortly describe it in Section 11). Note only here that in planar case JK- result is reduced to Theorem RKC.

The author has begun to consider harmonic functions in the space roughly since 2006 trying to generalize theory in the plane, cf [28, 3, 29, 22, 1, 31.

He realized some differences between theory in the plane and space and some difficulties to develop the space theory. It was observed that gradient mappings of harmonic functions are good candidate for generalization of the planar theory to space outlining some ideas and asking several open problems on the Belgrade Analysis seminar. Having studied Iwaniec's lecture [1] recently the author has found an additional motivation to investigate in this direction. For the present state see also the recent arXiv papers of Astala-Manojlović [5], Božin-Mateljević [ [6] and Mateljević [32.

Suppose that $F$ is mapping from a domain $G \subset \mathbb{R}^{n}$ (in particular, from the unit ball $\mathbb{B} \subset \mathbb{R}^{n}$ ) onto a bounded convex domain $D=F(G)$. To every $a \in \partial D$ 
we associate a nonnegative function $u=\underline{u}_{a}=\underline{F}^{a}$. Since $D$ is convex, for $a \in \partial D$, there is a supporting hyper-plane (a subspace of dimension $n-1) \Lambda_{a}$ defined by $\Lambda_{a}=\left\{w \in \mathbb{R}^{n}:\left(w-a, n_{a}\right)=0\right\}$, where $n=n_{a} \in T_{a} \mathbb{R}^{n}$ is a unit vector such that $\left(w-a, n_{a}\right) \geq 0$ for every $w \in \bar{D}$. Define $u(z)=\underline{F}^{a}(z)=\left(F(z)-a, n_{a}\right)$, cf. 25, 26, 27, 17. Our approuch here is also based on function $\underline{F}^{a}$.

We provide explicit interior lower bounds on the Jacobian in terms of the regularity of the domains and the boundary map in Section 11 For the convenience of the reader in Section 4 we mainly collect some results which we used in Section 1 and give a few additional results.

In Section 5, we outline short review of results from [14 for Harmonic Maps Between surfaces concerning lower bounds on the Jacobian, and the existence of harmonic diffeomorphisms which solve a Dirichlet problem.

In Section 2, estimates for the modulus of the derivatives of harmonic univalent mappings in space are given.

In Section 3 we generalize and develop the arguments used in planar theory of harmonic mappings to gradient mappings of harmonic functions in domains of $\mathbb{R}^{3}$. For example, we can consider the proof of Theorem 3.2 (which is not based on an approximation argument) as a suitable generalization of the proof of Theorem 1.3

\section{Estimates For the modulus of the DERIVATIVES OF HARMONiC UNIVALENT PLANAR MAPPINGS FROM BELOW}

1.1. For univalent harmonic maps between surfaces, estimates of Jacobian from below in terms of the geometric data involved are given in Jost 14 (see Corollary 8.1, Theorem 8.1) and for univalent euclidean harmonic maps in [17, 20, 27, 31. In this subsection, we consider convex codomains and give short review of a few result from [26, 25]. It seems that Theorem 1.3 is a new result. For a function $h$, we use notation $\partial h=\frac{1}{2}\left(h_{x}^{\prime}-i h_{y}^{\prime}\right)$ and $\bar{\partial} h=\frac{1}{2}\left(h_{x}^{\prime}+i h_{y}^{\prime}\right)$; we also use notations $D h$ and $\bar{D} h$ instead of $\partial h$ and $\bar{\partial} h$ respectively when it seems convenient.

Throughout this paper, if $h$ is a complex harmonic function on simple connected planar domain, we will write $h$ in the form $h=f+\bar{g}$, where $f$ and $g$ are holomorphic. Note that every complex valued harmonic function $h$ on simply connected domain $D$ is of this form.

Recall by $\mathbb{D}$ we denote the unit disc and $\mathbb{T}=\partial \mathbb{D}$ we denote the unit circle, and we use notation $z=r e^{i \theta}$. For a function $h$ we denote by $h_{r}^{\prime}, h_{x}^{\prime}$ and $h_{y}^{\prime}$ (or sometimes by $\partial_{r} h, \partial_{x} h$ and $\partial_{x} h$ ) partial derivatives with respect to $r, x$ and $y$ respectively. Let $h=f+\bar{g}$ be harmonic, where $f$ and $g$ are analytic. Then $\partial h=f^{\prime}, h_{r}^{\prime}=f_{r}^{\prime}+\overline{g_{r}^{\prime}}, f_{r}^{\prime}=f^{\prime}(z) e^{i \theta}$ and $J_{h}=\left|f^{\prime}\right|^{2}-\left|g^{\prime}\right|^{2}$. If $h$ is univalent, then $\left|g^{\prime}\right|<\left|f^{\prime}\right|$ and therefore $\left|h_{r}^{\prime}\right| \leq\left|f_{r}^{\prime}\right|+\left|g_{r}^{\prime}\right|$ and $\left|h_{r}^{\prime}\right|<2\left|f^{\prime}\right|$.

Theorem 1.1 (26]). Suppose that

(a) $h$ is a euclidean harmonic mapping from $\mathbb{D}$ onto a bounded convex domain $D=h(\mathbb{D})$, which contains the disc $B\left(h(0) ; R_{0}\right)$. Then

(i.1) $d(h(z), \partial D) \geq(1-|z|) R_{0} / 2, \quad z \in \mathbb{D}$.

(i.2) Suppose that $\omega=h^{*}\left(e^{i \theta}\right)$ and $h_{r}^{*}=h_{r}^{\prime}\left(e^{i \theta}\right)$ exist at a point $e^{i \theta} \in \mathbb{T}$, and there exists the unit inner normal $n=n_{\omega}$ at $\omega=h^{*}\left(e^{i \theta}\right)$ with respect to $\partial D$.

Then $\left(h_{r}^{*}, n\right) \geq c_{0}$, where $c_{0}=\frac{R_{0}}{2}$.

(i.3) In addition to the hypothesis stated in the item i.2), suppose that $h_{b}^{\prime}$ exists at 
the point $e^{i \theta}$. Then $\left|J_{h}\right|=\left|\left(h_{r}^{*}, N\right)\right|=\left|\left(h_{r}^{*}, n\right)\right||N| \geq c_{0}|N|$, where $N=i h_{b}^{\prime}$ and the Jacobian is computed at the point $e^{i \theta}$ with respect to the polar coordinates.

(i.4) If in addition to the hypothesis (a) suppose that $h$ is an euclidean univalent harmonic mapping from an open set $G$ which contains $\overline{\mathbb{D}}$. Then $\left|f^{\prime}\right| \geq \frac{R}{4}$ on $\mathbb{D}$.

A generalization of this result to several variables has been communicated at Analysis Belgrade Seminar, cf. [28].

Note that $(i .4)$ is a corollary of $(i .2)$.

Outline of proof of (i.1). To every $a \in \partial D$ we associate a nonnegative harmonic function $u=u_{a}$. Since $D$ is convex, for $a \in \partial D$, there is a supporting line $\Lambda_{a}$ defined by $\left(w-a, n_{a}\right)=0$, where $n=n_{a}$ is a unimodular complex number such that $\left(w-a, n_{a}\right) \geq 0$ for every $w \in \bar{D}$. Define $u(z)=\left(h(z)-a, n_{a}\right)$ and $d_{a}=d\left(h(0), \Lambda_{a}\right)$. Then $u(0)=\left(h(0)-a, n_{a}\right)=d\left(h(0), \Lambda_{a}\right)$ and therefore, by the mean value theorem,

$$
\frac{1}{2 \pi} \int_{0}^{2 \pi} u\left(e^{i t}\right) d t=u(0)=d_{a}=d\left(h(0), \Lambda_{a}\right) .
$$

Since $u=u_{a}$ is a nonnegative harmonic function, for $z=r e^{i \varphi} \in \mathbb{D}$, we obtain

$$
u(z) \geq \frac{1-r}{1+r} \frac{1}{2 \pi} \int_{0}^{2 \pi} u\left(e^{i t}\right) d t .
$$

Hence $u\left(r e^{i \varphi}\right) \geq d_{a}(1-r) / 2$, and therefore $|h(z)-a| \geq d_{a}(1-r) / 2 \geq(1-r) R_{0} / 2$. Thus $|h(z)-a| \geq(1-r) R_{0} / 2$ for every $a \in \partial D$ and therefore we obtain (1): $d(h(z), \partial D) \geq(1-r) R_{0} / 2$.

Note that if $D$ is a convex domain, then in general for $b \in \partial D$ there is no inner normal. However, there is a supporting line $\Lambda_{b}$ defined by $\left(w-b, n_{b}\right)=0$, where $n=n_{b}$ is a unimodular complex number such that $\left(w-b, n_{b}\right) \geq 0$ for every $w \in \bar{D}$.

Note that proof of theorem can also be based on Harnack's theorem (see also [40, Lemma 15.3.7) or Hopf Lemma.

We use the notation $\lambda_{f}=l_{f}(z)=|\partial f(z)|-|\bar{\partial} f(z)|$ and $\Lambda_{f}(z)=|\partial f(z)|+$ $|\bar{\partial} f(z)|$, if $\partial f(z)$ and $\bar{\partial} f(z)$ exist.

Theorem 1.2 ([25]). (ii.1) Suppose that $h=f+\bar{g}$ is a Euclidean orientation preserving harmonic mapping from $\mathbb{D}$ onto bounded convex domain $D=h(\mathbb{D})$, which contains a disc $B\left(h(0) ; R_{0}\right)$. Then $\left|f^{\prime}\right| \geq R_{0} / 4$ on $\mathbb{D}$.

(ii.2) Suppose, in addition, that $h$ is qc. Then $l_{h} \geq(1-k)\left|f^{\prime}\right| \geq(1-k) R_{0} / 4$ on $\mathbb{D}$ (ii.3) In particular, $h^{-1}$ is Lipschitz.

A proof of the theorem can be based on Theorem 1.1 and

(b): the approximation of a convex domain with smooth convex domains,

which is based on the hereditary property of convex functions: if an analytic function maps the unit disk univalently onto a convex domain, then it also maps each concentric subdisk onto a convex domain. Now we outline an approximation argument for convex domain $G$. Let $\phi$ be conformal mapping of $\mathbb{D}$ onto $G, \phi^{\prime}(0)>0$, $G_{n}=\phi\left(r_{n} \mathbb{D}\right), r_{n}=\frac{n}{n+1}, D_{n}=h^{-1}\left(G_{n}\right)$; and $\varphi_{n}$ conformal mapping of $\mathbb{D}$ onto $D_{n}$, $\varphi_{n}(0)=0, \varphi_{n}^{\prime}(0)>0$ and $h_{n}=h \circ \varphi_{n}$. Since $D_{n} \subset D_{n+1}$ and $\cup D_{n}=\mathbb{D}$, we can apply the Carathéodory theorem; $\varphi_{n}$ tends to $z$, uniformly on compacts, whence $\varphi_{n}^{\prime}(z) \rightarrow 1(n \rightarrow \infty)$. By hereditary property $G_{n}$ is convex.

Since the boundary of $D_{n}$ is an analytic Jordan curve, the mapping $\varphi_{n}$ can be continued analytically across $\mathbb{T}$, which implies that $h_{n}$ has a harmonic extension 
across $\mathbb{T}$. An application of Theorem $1.1(i .4)$ to $h_{n}$ gives the proof.

Example 1. (ii.4) $f(z)=(z-1)^{2}$ is univalent on $\mathbb{D}$. Since $f^{\prime}(z)=2(z-1)$ it follows that $f^{\prime}(z)$ tends 0 if $z$ tends 1 . This example shows that we can not drop the hypothesis that $f(\mathbb{D})$ is a convex domain in Theorem 1.1 (i.4).

(ii.5) $g(z)=\sqrt{z+1}$ is univalent on $\mathbb{D}$ is not Lipshitz on $\mathbb{D}$.

(ii.6) $g^{-1}(w)=w^{2}-1$ and $\left(g^{-1}\right)^{\prime}(w)=2 w$ tends to 0 if $w \in g(\mathbb{D})$ tends to 0 .

1.2. Hall, see [7 p. 66-68, proved the following:

Lemma 1.1 (Hall lemma). (ii.7) For all harmonic univalent mappings $f$ of the unit disk onto itself with $f(0)=0$, $\left|a_{1}\right|^{2}+\left|b_{1}\right|^{2} \geq c_{0}=\frac{27}{4 \pi^{2}}$

where $a_{1}=D f(0), b_{1}=\bar{D} f(0)$ and $c_{0}=\frac{27}{4 \pi^{2}}=0.6839 \ldots$.

(ii.8) If in addition $f$ is orientation preserving, then $\left|a_{1}\right| \geq \sigma_{0}$, where $\sigma_{0}=\frac{3 \sqrt{3}}{2 \sqrt{2} \pi}$.

Hence, one can derive:

(I0) There is a constant $c>0$ such that if $h$ is harmonic planar mapping and $B(h x, R) \subset h(B(x, r))$, then $r \Lambda_{h}(x) \geq c R$.

Now we give another proof and generalization of the part (ii.3) of Theorem 1.2 . which is not based on the approximation of a convex domain with smooth convex domains.

Theorem 1.3. Suppose that (a.1): $D$ and $D_{*}$ are simply-connected hyperbolic domains in $\mathbb{R}^{2}$ with non-empty boundary and that $f$ is a euclidean harmonic univalent mapping from $D$ onto $D_{*}$. Then

(iii.1) $d \Lambda_{f} \succeq d_{*}$ on $D$, where $w=f(z), d=\operatorname{dist}(z, \partial D)$ and $d_{*}(w)=\operatorname{dist}\left(w, \partial D_{*}\right)$. (iii.2) If in addition to hypothesis (a.1) we suppose that (a.2): $D$ is a $C^{1, \alpha}, 0<\alpha<$ 1 , domain and $D_{*}$ is convex bounded domain, then there is a constant $c>0$ such that $\Lambda_{f} \succeq c$ on $D$.

By Theorem 4.7(Kellogg) we can reduce the proof to the case $D=\mathbb{D}$.

Proof. Let $z \in D$ and $\phi_{0}$ conformal of $\mathbb{B}$ onto $D$ such that $\phi_{0}(0)=z$ and $F=h \circ \phi_{0}$. Since $\Lambda_{F}=\Lambda_{f}\left|\phi_{0}^{\prime}\right|$, by (I0) version of Hall lemma, $\Lambda_{F} \succeq d_{*},\left|\phi_{0}^{\prime}\right| \asymp d$. Hence we find (iii.1): $d \Lambda_{f} \succeq d_{*}$.

Now let $z=\phi\left(z^{\prime}\right)$ be a conformal of $\mathbb{B}$ onto $D$ and $d^{\prime}=\operatorname{dist}\left(z^{\prime}\right)$. Then $d^{\prime}\left|\phi^{\prime}\right| \asymp d$ and by Theorem 4.7(Kellogg) (iii.3): $d^{\prime} \asymp d$.

Since $D_{*}$ convex, $d_{*} \succeq d^{\prime}$ and therefore by (iii.3) we find $d_{*} \succeq d$. Hence by (iii.1) $\Lambda_{f} \succeq c$ on $D$.

The following result is an immediate corollary of Theorem 1.3

Theorem 1.4. If in addition to hypothesis (a.1) and (a.2) of Theorem $1.3 f$ is qc, then $f^{-1}$ is Lipschitz on $D_{*}$.

Corollary 1. In particular, if $f$ is conformal, then $f^{-1}$ is Lipschitz on $D_{*}$.

Proof. Using that $f$ is qc, it follows that $\lambda_{f} \succeq \Lambda_{f} \succeq c$ and the rest of the proof is routine.

Let $G$ be simply connected hyperbolic planar domain and $\rho=\rho_{G}^{\text {hyp }}$ hyperbolic density; we also use short notation $\rho_{h y p}=\rho_{G}$. Using the uniformization theorem, one can define hyperbolic density for a hyperbolic planar domain. 
If $G$ is a planar domain with non-empty boundary, $f$ a $C^{1}$ complex valued mapping $d=d_{G}$ and $d_{*}=d_{f(G)}$, we define $H:=|\partial f|_{h y p}^{q v}=\frac{d(z)}{d_{*}(f(z))}\left|f_{z}\right|$. In addition if $G$ is hyperbolic planar domain, $\rho=\rho_{G}$ and $\rho_{*}=\rho_{f(G)}$, we define $H:=|\partial f|_{h y p}=\frac{\rho_{*}(f(z))}{\rho(z))}\left|f_{z}\right|$.

Theorem 1.5. If $G$ is simply connected, then

(iv.1) $d_{G}(w) \leq \rho_{G}^{-1} \leq 8 d_{G}(w)$.

There is an absolute constant c such that under the hypothesis (a.1) of Theorem 1.3 if $f$ in addition orientation preserving, we have

(iv.2) $H:=|\partial f|_{h y p}^{q v}=\frac{d(z)}{d_{*}(f(z))}\left|f_{z}\right| \geq c$.

(iv.3) $H:=|\partial f|_{h y p} \geq c / 8$.

Proof. Let $\phi: \mathbb{D} \rightarrow G$ be conformal. Then $\rho(w)\left|\phi^{\prime}(z)\right|=\rho(z)$ and $d_{G}(w) \leq$ $d_{\mathbb{D}}(z)\left|\phi^{\prime}(z)\right| \leq 4 d_{G}(w)$. Hence $d_{G}(w) \leq \rho_{G}^{-1} \leq 8 d_{G}(w)$.

Set $w=\phi(z), \zeta=f(w)$ and $F=f \circ \phi$. Since $F_{z}=f_{w} \phi^{\prime}(z)$, by an application of Hall lemma, (ii.8), to $F$, we find $\left|f_{w}(w)\right|\left|\phi^{\prime}(z)\right| \geq d_{*} \sigma_{0}$. If $w \in G$, we can choose a conformal mapping $\phi_{0}: \mathbb{D} \rightarrow G$ such that $w=\phi_{0}(0)$. Then $\left|\phi_{0}^{\prime}(0)\right| \leq 4 d$ and therefore $4 d\left|f_{w}(w)\right| \geq d_{*} \sigma_{0}$. Hence we get (iv.2) with $c=\sigma_{0} / 4$.

The part (iv.3) has also been proved by Kalaj.

1.3. The minimum principle for the Jacobian. (I1) Let $h: \Omega \rightarrow \mathbb{C}$ be a harmonic map whose Jacobian determinant $J=\left|h_{z}\right|^{2}-\left|h_{\bar{z}}\right|^{2}$ is positive everywhere in $\Omega$. Then $-\ln J$ is subharmonic; More precisely, cf. [11, 12,

$$
-\frac{1}{4} \Delta \ln J=-(\ln J)_{z \bar{z}}=\frac{\left|h_{z z} \overline{h_{\bar{z}}}-\overline{h_{\bar{z}}} h_{z}\right|^{2}}{J^{2}} .
$$

Note that in [?] it is proved previously that

(I2) $X=\log \frac{1}{J_{h}}$ is a subharmonic function.

We left to the reader to check the following fact (I3-I6):

If $F$ is an analytic function, then $|F|_{z \bar{z}}^{2}=\left|F^{\prime}\right|^{2}$. Hence

(I3) $J(h)_{z}=f^{\prime \prime} \overline{f^{\prime}}-g^{\prime \prime} \overline{g^{\prime}}, J(h)_{z \bar{z}}=\left|f^{\prime \prime}\right|^{2}-\left|g^{\prime \prime}\right|^{2}$.

(I4) In general, $J(h)$ nether subharmonic nor superharmonic.

If $\tau: D \rightarrow I, I=(a, b), \chi: I \rightarrow R$, then

(I5) $(\chi \circ \tau)_{z \bar{z}}=\left(\chi^{\prime \prime} \circ \tau\right) \tau_{\bar{z}} \tau_{z}+\left(\chi^{\prime} \circ \tau\right) \tau_{z \bar{z}}=\left(\chi^{\prime \prime} \circ \tau\right)\left|\tau_{z}\right|^{2}+\left(\chi^{\prime} \circ \tau\right) \tau_{z \bar{z}}$.

If we set $\chi(x)=x^{-1}$ and $\tau=J$, we find

(I6) $-\left(J^{-1}\right)_{z \bar{z}} J^{3}=\left|\tau_{z}\right|^{2}+|B+C|^{2}$.

If we set $\chi=\log , \tau=J, B=f^{\prime} g^{\prime \prime}$ and $C=g^{\prime} f^{\prime \prime}$, then

(I7) $-(\ln J)_{z \bar{z}} J^{2}=|B+C|^{2}$.

(I8) Suppose that $F$ and $H$ are analytic function in a domain $G$ such that $|F|^{2}=m_{0}+|H|^{2}$ on $G$, where $m_{0}$ is a positive constant. Then $F_{z} \bar{F}=H_{z} \bar{H}$ and therefore

$H^{\prime} / F^{\prime}=\bar{F} / \bar{H}$. Hence $H^{\prime} / F^{\prime}=a_{0}$, where $a_{0}$ is a constant and therefore $H / F=$ $a_{0} z+a_{1}$.

$|F|^{2}=m_{0}+\left|a_{0} z+a_{1}\right|^{2}|F|^{2}$ on $G$. Without loss of generality we can suppose that $0 \in G$ and that $F=b_{0}+b_{n} z^{n}+o\left(z^{n}\right), n \geq 1$.

This leads to a contradiction and so $F=b_{0}$ and therefore $H=c_{0}$ on $G$, where $b_{0}$ and $c_{0}$ are constants. 
Proposition 1.1. (a.2) If $h$ is harmonic on $U$ and $J(h)$ attains minimum (different from 0 ) at interior point a, then $J(h)$ is constant function, and $h$ is affine.

(b.2) Functions $\log \frac{1}{J_{h}}$ and $\frac{1}{J_{h}}$ are subharmonic function.

(c.2) In particular, $\log J_{h}$ is a superharmonic function.

Corollary 2 (Minimum Principle). Let $h: \Omega \rightarrow \mathbb{C}$ be a harmonic map whose Jacobian determinant $J$ is positive everywhere in $\Omega$. Then $\inf _{F} J \geq$ inf $_{\partial F} J$ for every compact $F \subset \Omega$.

Example $h=f+\bar{g}$, where $f=4 z, g=z^{2} / 2$, shows that that analog statement is not valid for maximum; $J=16-\left(x^{2}+y^{2}\right)$ attains maximum 16 at $(0,0)$.

In general, min $J$ is attained at the boundary.

Proof of (a.2). Suppose that $h$ is orientation preserving and $J(h)=\left|f^{\prime}\right|^{2}-\left|g^{\prime}\right|^{2}$ attains min (different from 0) at interior point $a$; then $\left|f^{\prime}\right|^{2}-\left|g^{\prime}\right|^{2} \geq J(h, a)=m$, $\left|f^{\prime}\right|^{2} \geq m+\left|g^{\prime}\right|^{2}$ and therefore $1 \geq s(z)$, where

$$
s=\frac{m}{\left|f^{\prime}\right|^{2}}+\frac{\left|g^{\prime}\right|^{2}}{\left|f^{\prime}\right|^{2}} .
$$

Since $s$ is subharmonic and $s(a)=1, s$ is a constant, ie. $s=1$. Hence $\left|f^{\prime}\right|^{2}=$ $m+\left|g^{\prime}\right|^{2}$, ie. $J=m$. By (I8), $f^{\prime}$ and $g^{\prime}$ are constant functions and therefore $h$ is affine.

Proof of (b.2). Hence, since exp is a convex increasing function, it follows that $\exp \circ X=\frac{1}{J_{h}}$ is also a subharmonic function.

Although $\chi(x)=e^{-x}$ is convex the conclusion that $\chi \circ X=J(h)$ is a subharmonic function is not true in general. Note that here $\chi$ is a decreasing function. In general, the minimum modulus principle for complex-valued harmonic functions is not valid; see the following examples:

Example 2. 1. If $f(z)=x+i$, then $|f(z)|^{2}=x^{2}+1$ and $|f|$ attains minimum which is 1 for every points on $y$ axis

2. If $f_{c}(z)=x+i\left(x^{2}-y^{2}+c\right)$, then $J_{f}=-2 y$. Let $d: \mathbb{C} \rightarrow R$ is given by $d(z)=|z|$, $z \in \mathbb{C}, g=f_{-1}, C(x)=x+i\left(x^{2}-1\right)$ and $D=\left\{(x, y): y<x^{2}-1\right\}$. Since $g(\mathbb{C})=D$ and $0 \notin D$, then $d$ attains minimum on $\operatorname{tr}(C)$ at some point $w_{0}$ and there is a real point $x_{0}$ such that $g\left(x_{0}\right)=w_{0}, g$ maps $\mathbb{C}$ onto $D$ and $|g|$ attains minimum at $x_{0}$.

Let $c<0$ and $D_{c}=\left\{(x, y): y<x^{2}-|c|\right\}$. Then $f_{c}(\mathbb{C})=D_{c}$ and $0 \notin D_{c}$. At first sight someone can guess that $\left|f_{c}(0)\right|=|c|$ is the minimum value for $\left|f_{c}\right|$ if $c<0$ ? We leave to the interested reader to show that $|c|$ is not the minimum value.

1.4. Outline of proof of Theorem RCK given in 11, 12. For $f: \mathbb{T} \rightarrow \mathbb{C}$, we define $\underline{\mathrm{f}}$ on $[0,2 \pi]$ by $\underline{\mathrm{f}}(t)=f\left(e^{i t}\right)$. Let $\gamma$ be a closed Jordan curve, $G=\operatorname{Int}(\gamma)$, $f_{0}: S^{1} \stackrel{\text { onto }}{\longrightarrow} \gamma$ a monotone map and $F=P\left[f_{0}\right]$.

Theorem 1.6 (T. Radó H. Kneser G. Choquet, Theorem RKC). If G is convex, then $F$ is a homeomorphism of $\mathbb{D}$ onto $G$.

Iwaniec-Onninen [11, 12] presented a new analytic proof of RKC-Theorem. The approach is based on the following steps.

c1) Prove the theorem if $f_{0}$ is diffeomorphism and $G$ is a smooth strictly convex domain, using the minimum principle for the Jacobian determinant and explicit interior lower bounds on the Jacobian in terms of the regularity of the domains and the boundary map. 
c2) Let $G$ be still a smooth strictly convex domain, but $f: S^{1} \stackrel{\text { onto }}{\longrightarrow} \gamma$ an arbitrary monotone map. This map can easily be shown to be a uniform limit of diffeomorphisms. Now the Poisson extensions $F_{j}$ are harmonic diffeomorphisms in $\mathbb{D}$, converging uniformly on $\overline{\mathbb{D}}$ to $F$ and $J_{F}>0$, cf also [20, 28].

c3) the approximation of a convex domain with smooth convex domains.

c4) there is a conformal map $\phi$ of $\mathbb{D}$ onto $G$; by variation of boundary values deform this conformal map into a harmonic diffeomorphism.

It is convenient to give kinematic description of $f: \mathbb{S}^{1} \stackrel{\text { onto }}{\longrightarrow} \gamma$, to view it as motion of an object along $\gamma$ in which $\mathbb{S}^{1}$ is labeled as a clock. As time runs from 0 to $2 \pi$ the motion $t \rightarrow \underline{\mathrm{f}}(t)$ begins at the point $\underline{\mathrm{f}}(0)=0$ and terminates at the same point $\underline{\mathrm{f}}(2 \pi)=0$. The velocity vector $v(t)=\underline{\mathrm{f}}^{\prime}(t)$ is tangent to $\gamma$ at $s=s(t)=\int_{0}^{t}|v(\tau)| d \tau$. We call $|v(t)|$ the speed. Let $z=z(s)$ be the length parametrization of $\gamma$. Since $\left|z^{\prime}(s)\right|=1$, we have $z(s)=e^{i \varphi(s)}$, where $\varphi(s)$ referred to as the tangential angle, is uniquely determined by the arc parameter $s$ because $G$ is smooth and strictly convex. The derivative is exactly the curvature of $\gamma$; that is $\kappa(s)=\varphi^{\prime}(s)$. The speed $|v(t)|$, being positive, uniquely represents unique diffeomorphism $f: S^{1} \stackrel{\text { onto }}{\longrightarrow} \gamma$. An explicit formula for $f$ involves the curvature of $\gamma$.

Theorem 1.7 ([11, 12]). Let $f: S^{1} \stackrel{\text { onto }}{\longrightarrow} \gamma$ be a $C^{\infty}$-difeomorphism and $F$ : $\mathbb{D} \rightarrow C$ its continuous harmonic extension. Then $F: \mathbb{D} \stackrel{\text { onto }}{\longrightarrow} G$ is a $C^{1}$-smooth diffeomorphism whose Jacobian determinant satisfies:

$$
J_{F} \geq \frac{k m^{3}}{2 \pi K M} \text { everywhere in } \mathbb{D}
$$

provided $0<k \leq \min \kappa(s) \leq \max \kappa(s) \leq K$ and $0<m \leq \min v(t) \leq \max v(t)(s) \leq$ $M$, where $v(t)=\left|\underline{f}^{\prime}(t)\right|$.

In [11, 12 the strategy is used to prove first The Lower Bound of the Jacobian along $\mathbb{S}^{1}$. Then the proof is reduced to showing inequality (1.1) at the boundary of the disk, as one may have expected from the Minimum Principle.

1.5. We also can use Theorem 1.8 below, which yields appriori estimate, instead of Theorem 1.7 in the procedure of proof of Theorem RKC.

Let $A(\gamma)$ be the family of $C^{1}$-difeomorphism $f: \mathbb{S}^{1} \stackrel{\text { onto }}{\longrightarrow} \gamma$. Set $v(t)=v_{f}(t)=$ $\left|\underline{f^{\prime}}(t)\right|$.

Let $d$ be diameter of $G, b \in G, B=B\left(b ; d / 2, \gamma_{1}\right.$ the part of $\gamma$ out of $B$ and $I=\left\{t: f(t) \in \operatorname{tr}\left(\gamma_{1}\right)\right\}$. Then $d / 2 \leq\left|\gamma_{1}\right| \leq M|I|$.

Theorem 1.8. Let $f \in A(\gamma)$ and $0<m \leq \min v(t) \leq \max v(t)(s) \leq M$. Then

$$
J_{F} \geq \frac{d m}{8 \pi M} \text { on } \mathbb{D} \text {. }
$$

Using approuch outlined in [26, 27, we can prove

Lemma 1.2. The inequality (1.2) holds everywhere in $\mathbb{S}^{1}$.

Naturally, the interior estimate at (1.2) would follow from the already established estimate at the boundary (via the minimum principle) if we knew that the Jacobian of $F$ was positive in $\mathbb{D}$. 
But, by Theorem RKC the Jacobian of $F$ is positive in $\mathbb{D}$. Theorem 1.9 below yields better estimate. Using Theorem 1.1, the part (i.3), and the minimum principle for Jacobian one can derive:

Theorem 1.9. $\mathrm{d} 1)$ Let $\Omega$ be a convex Jordan domain, $f: \mathbb{T} \rightarrow \partial \Omega$ absolutely continuous homeomorphism which preserves orientation, and let $w=P[f]$ be a harmonic function between the unit disk and $\Omega$, such that $w(0)=0$,

d2) $\left|\underline{f}^{\prime}(t)\right| \geq m$, for almost every $0 \leq t \leq 2 \pi$,

d3) $\underline{f}^{\prime}$ is Dini's continuous.

Then the following results hold (iv.1): $J_{w}(z)>m \operatorname{dist}(0, \partial \Omega) / 2$, for every $z \in \mathbb{D}$, and

(iv.2) $w$ is bi-Lipschitz.

Let $X$ be a compact subset of a metric space with metric $d_{1}\left(\right.$ such as $\mathbb{R}^{n}$ ) and let $f: X \rightarrow Y$ be a function from $X$ into another metric space $Y$ with metric $d_{2}$. The modulus of continuity of $f$ is $\omega_{f}(t)=\sup _{d_{1}(x, y) \leq t} d_{2}(f(x), f(y)), t>0$. The function $f$ is called Dini-continuous if

$$
\int_{0}^{1} \frac{\omega_{f}(t)}{t} d t<\infty
$$

Dini continuity is a refinement of continuity. Every Dini continuous function is continuous. Every Lipschitz continuous function is Dini continuous. Note that under the above hypothesis $\underline{f}^{\prime}$ has continuous extension to $[0,2 \pi]$ and partial derivatives of $w$ have continuous extension to $\overline{\mathbb{D}}$ and one can show that $w$ is bi-Lipschitz. We can use an aproximation argument to prove (iv.1) for $C^{1, \alpha}$ domains $\Omega$ without the hypothesis d3). Moreover, an application of $H^{p}$ theory shows that the following result, due to Kalaj, holds in general:

Theorem 1.10 (Theorem 2.8, Corollary 2.9 [19]). Under hypothesis $\mathrm{d} 1$ ) and $\mathrm{d} 2$ ) of Theorem 1.9, $J_{w}^{*}$ exsists a.e. on $\mathbb{T}$ and (iv.1) holds.

Under hypothesis d1) of Theorem 1.9, Theorem RKC states that (v.1) $J_{w}>0$ on $\mathbb{D}$.

Question 1. Can we modify approach in [11, 12] to give analytic proof of Theorem 1.10 (of course without appeal to Theorem RKC (moreprecisely to (v.1))?

\section{Estimates FOR the MOdulus of the DERIVATIVES OF HARMONiC UNIVALENT MAPPINGS IN SPACE}

Definition 2.1. Let $G$ be a subset of the Euclidean space $\mathbb{R}^{n}$ and let

$$
\varphi: G \rightarrow \mathbb{R} \cup\{+\infty\}
$$

be an lower semi-continuous function. Then, $\varphi$ is called $q$ - superharmonic, $0<q \leq$ 1 , if for any closed ball $\overline{B(x, r)}$ of center $x$ and radius $r$ contained in $G$ and every real-valued continuous function $h$ on $\overline{B(x, r)}$ that is harmonic in $B(x, r)$ and satisfies $\varphi(y) \geq q h(y)$ for all $y$ on the boundary $\partial B(x, r)$ of $B(x, r)$ we have $\varphi(y) \geq h(y)$ for all $y \in B(x, r)$. If $q=1$ we say superharmonic instead of 1- superharmonic.

Definition 2.2. Let $G$ be a domain in $\mathbb{R}^{n}$. Suppose that $f: G \rightarrow \mathbb{R}^{n}$ is a $C^{1}$ function and there is a constant $c>0$ such that for every $x \in G$

(a) if $B(x, r) \subset G$ and $B(f x, R) \subset f(B(x, r))$, then $r \Lambda_{f}(x) \geq c R$.

(a') if $B(f x, R) \subset f(\underline{B}(x, r))$, then $r \Lambda_{f}(x) \geq c R$. 
We say that $f$ has $H$-property (respectively weak $H$-property) if (a) (respectively (a')) holds.

By Hall lemma planar euclidean harmonic mappings have $H$-property.

We say that $\underline{F} \subset H_{Q} C_{K}\left(G, G^{\prime}\right)$ has $H$-property if $F$ is closed with respect to uniform convergence, and for $f \in \underline{F}, J(f)$ has no zeros in $G$.

If $\underline{F} \subset H Q C_{K}\left(G, G^{\prime}\right)$ has $H$-property, then $f \in \underline{F}$ has weak $H$-property.

By Lemma 3.1 there is a constant $c>0$ such that $d(x) \Lambda_{f}(x) \geq c d(f x), x \in G$.

Lemma 2.1. Let $F_{K}$ be a family of harmonic K-qc mapping $f: \mathbb{B} \rightarrow R^{n}$ such that for $f \in F_{K}, J(f)$ has no zeros, $F_{K}$ is closed with respect to uniform convergence, $f(\mathbb{B}) \supset \mathbb{B}$ and $f(0)=0$. Then there is a constant $c>0$ such that if $f \in F_{K}$ is harmonic K-qc mapping $f(\mathbb{B}) \supset \mathbb{B}, f(0)=0$, then $\Lambda_{f}(0) \geq c$.

Contrary there is a sequence $f_{n} \in F_{K}$ such that $\Lambda_{f_{n}}(0) \rightarrow 0$. Sequence $f_{n}$ forms a normal family and there is a subsequence of $f_{n}$ which converges uniformly to a limit $f_{0} \in F_{K}$; this is a contradiction.

Suppose that $F$ is mapping from the unit ball $\mathbb{B} \subset \mathbb{R}^{n}$ into $\mathbb{R}^{m}$ and suppose that $\omega=F^{*}(x)$ and $\left(\partial_{r} F\right)^{*}(x)$ exist at a point $x \in \mathbb{S}$. Then

(A1) $F_{r}^{\prime}(x)$ exists and $\left(\partial_{r} F\right)^{*}(x)=F_{r}^{\prime}(x)$.

Proof of $(A 1)$. By Lagrange theorem, there is $t_{k} \in[r, 1)$ such that $F_{k}(r x)-$ $F_{k}(x)=-\left(F_{k}\right)_{r}^{\prime}\left(t_{k} x\right)(1-r)$. Hence, since $\left(\partial_{r} h\right)^{*}(x)$ exist at a point $x \in \mathbb{S}$, if $r$ tends 1 , then $\left(F_{k}\right)_{r}^{\prime}\left(t_{k} x\right)$ tends $\left(F_{k}\right)_{r}^{\prime}(x)$.

Theorem 2.1. (a1) Suppose that $h$ is a euclidean harmonic mapping from the unit ball $\mathbb{B} \subset \mathbb{R}^{n}$ onto a bounded convex domain $D=h(\mathbb{B})$, which contains the ball $B\left(h(0) ; R_{0}\right)$. Then

(i.1) $d(h(z), \partial D) \geq(1-|z|) \bar{c}_{n} R_{0}, \quad z \in \mathbb{B}$, where $\bar{c}_{n}=\frac{1}{2^{n-1}}$.

(i.2) For every $x \in \mathbb{S}$ and for $0<r<1$, there is $t \in[r, 1)$ such that $\left|h_{r}^{\prime}(t x)\right| \geq c_{0}$.

(i.3) If $h$ is $\mathrm{K}$-qc and (a2) $h$ has $H$-property or (a3) for some $k,\left|h_{x_{k}}^{\prime}\right|^{n}$ is $q$ - super harmonic, then $h$ is co-Lipschitz on $\mathbb{B}$.

(a4) Suppose, in addition, to (a1) that $h$ is $\mathrm{K}$-qc and $D$ is $C^{2}$ domain. Then (i.4) $h_{r}^{\prime}(x)$ exists and $\left(\partial_{r} h\right)^{*}(x)=h_{r}^{\prime}(x) \geq c_{0}$ for almost everywhere $x \in S$.

Proof of (i.1). To every $a \in \partial D$ we associate a nonnegative harmonic function $u=u_{a}$. Since $D$ is convex, for $a \in \partial D$, there is a supporting hyper-plane $\Lambda_{a}$ defined by $\left(w-a, n_{a}\right)=0$ where $n=n_{a} \in T_{a} \mathbb{R}^{n}$ is a unit vector such that $\left(w-a, n_{a}\right) \geq 0$ for every $w \in \bar{D}$. Define $u(z)=\left(h(z)-a, n_{a}\right)$ and $d_{a}=d\left(h(0), \Lambda_{a}\right)$. Then $u(0)=\left(h(0)-a, n_{a}\right)=d\left(h(0), \Lambda_{a}\right)$. Let $a_{0} \in \Lambda_{a}$ be the point such that $d_{a}=\left|h(0)-a_{0}\right|$. Then from geometric interpretation it is clear that $d_{a} \geq R_{0}$.

By Harnack's inequality, $\bar{c}_{n}(1-r) u(0) \leq u(x), x \in \mathbb{B}$ and $r=|x|$, where $\bar{c}_{n}=$ $\frac{1}{2^{n-1}}$. In particular, $\bar{c}_{n} d(x) R_{0} \leq u(x) \leq|h(x)-a|$ for every $a \in \partial D$. Hence, for a fixed $x, d_{h}(x)=\inf _{a \in \partial D}|h(x)-a| \geq \bar{c}_{n} R_{0} d(x)$ and therefore we obtain (i.1). then

Proof of (i.2). Set $u(z)=\left(h(z)-h(x), n_{h(x)}\right)$ and $c_{0}=\bar{c}_{n} R_{0}$. Since $u(x)=0$,

(i.5) for $x \in \mathbb{S}, u(r x)-u(x) \geq c_{0}(1-r)$.

By Lagrange theorem, there is $t \in[r, 1)$ such that $u(r x)-u(x)=u_{r}^{\prime}(t x)(1-r)$.

Proof of (i.3). Suppose for example (b1). By Theorems 2.1 and 2.2 $\underline{J}(x) \succeq \frac{d_{*}}{d} \succeq$ $c$ for every $x \in \mathbb{B}$. By (a3),

$$
\left|h_{x_{k}}^{\prime}(x)\right|^{n} \geq q \frac{1}{\left|\underline{B}_{x}\right|} \int_{\underline{B}_{x}}\left|h_{x_{k}}^{\prime}(z)\right|^{n} d z, x \in \mathbb{B} \text {. Hence }\left|h_{x_{k}}^{\prime}(x)\right| \succeq \underline{J}(x) \succeq c
$$


and $\lambda\left(h^{\prime}(x) \succeq c\right.$ and therefore $h$ is co-Lipschitz on $\mathbb{B}$.

Proof of (i.4). First, suppose that $h_{r}^{\prime}(x)$ exists for some $x \in \mathbb{S}$. By $(i .5)$, $\left(\frac{h(r x)-h^{*}(x)}{1-r}, n\right) \geq c_{0}$, where $n=n_{a}$ and $a=h^{*}(x)$. Hence, since $h_{r}^{\prime}(x)$ exists, it follows

(i.6) $\left(h_{r}^{\prime}(x), n\right) \geq \bar{c}_{n} R_{0}$.

By a result of D. Kalaj [21, partial derivatives of $h$ are bounded and therefore $h_{r}^{\prime}(x)$ exists for almost everywhere $x \in S$ and therefore by $(i .2)$ and (i.6), $\left(\partial_{r} h\right)^{*}(x)=h_{r}^{\prime}(x) \geq c_{0}$ for almost everywhere $x \in \mathbb{S}$.

In [22] it is proved the following theorem: a $K$ quasiconformal harmonic mapping of the unit ball $\mathbb{B}^{n}(n>2)$ onto itself is Euclidean bi-lipschitz, providing that $u(0)=0$ and that $K<2^{n-1}$, where $n$ is the dimension of the space. It is an extension of a similar result for hyperbolic harmonic mappings with respect to hyperbolic metric (see Tam and Wan, (1998)). The proof makes use of Möbius transformations in the space, and of a recent result which states that, harmonic quasiconformal self-mappings of the unit ball are Lipschitz continuous; this result first has been proved by the first author and then generalized also by the second author.

Introduce the quantity

$$
a_{f}(x)=a_{f, G}(x):=\exp \left(\frac{1}{n\left|B_{x}\right|} \int_{B_{x}} \log J_{f}(z) d z\right), x \in G,
$$

associated with a quasiconformal mapping $f: G \rightarrow f(G) \subset \mathbb{R}^{n}$; here $J_{f}$ is the Jacobian of $f$; while $\mathbf{B}_{x}=\mathbf{B}_{x, G}$ stands for the ball $B(x ; d(x, \partial G))$; and $\left|\mathbf{B}_{x}\right|$ for its volume. Astala and Gehring [4] observed that for certain distortion property of quasiconformal mappings the function $a_{f}$, defined the above, plays analogous role as $\left|f^{\prime}\right|$ when $n=2$ and $f$ is conformal; and they establish quasiconformal version of the well-know result due to Koebe, cited here as Lemma 2.2

Lemma 2.2. 4] Suppose that $G$ and $G^{\prime}$ are domains in $R^{n}:$ If $f: G \rightarrow G^{\prime}$ is $K$-quasiconformal, then

$$
\frac{1}{c} \frac{d\left(f(x), \partial G^{\prime}\right)}{d(x, \partial G)} \leq a_{f, G}(x) \leq c \frac{d\left(f(x), \partial G^{\prime}\right)}{d(x, \partial G)}, \quad x \in G,
$$

where $c$ is a constant which depends only on $K$ and $n$.

Our next result concerns the quantity

$$
\underline{E}_{f, G}(x):=\frac{1}{\left|\underline{B}_{x}\right|} \int_{\underline{B}_{x}} J_{f}(z) d V(z), x \in G,
$$

associated with a quasiconformal mapping $f: G \rightarrow f(G) \subset \mathbb{R}^{n}$; here $d V(z)=d z$ is the Euclidean volume element $d z_{1} d z_{2} \cdots d z_{n}$ and $z=\left(z_{1} \cdots z_{n}\right)$ and $J_{f}$ is the Jacobian of $f$; while $\underline{B}_{x}=\underline{B}_{x, G}$ stands for the ball $B(x, d(x, \partial G) / 2)$ and $\left|B_{x}\right|$ for its volume.

Define

$$
\underline{J}_{f}=\underline{J}_{f, G}=\sqrt[n]{\underline{E}_{f, G}} .
$$

Theorem 2.2 (31]). Suppose that $G$ and $G^{\prime}$ are domains in $\mathbb{R}^{n}:$ If $f: G \rightarrow G^{\prime}$ is $K$-quasiconformal, then 


$$
\frac{1}{c} \frac{d\left(f(x), \partial G^{\prime}\right)}{d(x, \partial G)} \leq \underline{J}_{f, G}(x) \leq c \frac{d\left(f(x), \partial G^{\prime}\right)}{d(x, \partial G)}, \quad x \in G,
$$

where $c$ is a constant which depends only on $K$ and $n$.

If $G$ and $G^{\prime}$ are domains in $\mathbb{R}^{n}$, by $Q C H\left(G, G^{\prime}\right)$ (respectively $Q C H_{K}\left(G, G^{\prime}\right)$ ) we denote the set of Euclidean harmonic quasiconformal mappings (respectively K-qc) of $G$ onto $G^{\prime}$.

If $D$ is a domain in $\mathbb{R}^{n}$, by $Q C H(D)$ we denote the set of Euclidean harmonic quasiconformal mappings of $D$ onto itself.

Definition 2.3. Let $Q_{H}(G)$ be a family of harmonic mappings $h$ from $G$ into $\mathbb{R}^{n}$ such that

(b1) $J(h)$ has no zeros in $G$, and

(b2) which is closed with respect to uniform convergence on compact subsets and

(b3) for every sequence $x_{n}$ which tends to a $x_{0} \in \partial G, h_{n} \in Q_{H}$, where $h_{n}(x)=$ $\frac{1}{d_{n}} h\left(\left(d_{n} x+x_{n}\right)\right)$ and $d_{n}=d\left(x_{n}\right)=\operatorname{dist}\left(x_{n}\right)$. If $G$ is the unit ball $\mathbb{B}$ we write $Q_{H}$ instead of $Q_{H}(G)$.

Using a criteria for normality of a qc family, one can establish criteria when a subfamily $Q$ of $Q C H(G)$, for which $K_{O}(f)<3^{n-1}$ for every $f \in Q$, is $Q_{H}(G)$ family.

Definition 2.4. Let $f: G \rightarrow G^{\prime}$ be a $C^{1}$ function. We say that $f$ has Jacobian non zero normal family-property if

(b1) $J(f)$ has no zeros in $G$, and

(b2) for every sequence $x_{n}$ which tends to a $x_{0} \in \partial G,\left(f_{n}\right)$ forms a normal family, where $f_{n}(x)=\frac{1}{d_{n}^{*}} f\left(\left(d_{n} x+x_{n}\right)\right)$, and $d_{n}=d\left(x_{n}\right)=\operatorname{dist}\left(x_{n}\right)$ and $d_{n}^{*}=d\left(f\left(x_{n}\right)\right)$ and

(b3) for every limit $f_{0}$ of $\left(f_{n}\right)$ in sense of the uniform convergence, $f_{0}$ is a $C^{1}$ function and $J\left(f_{0}\right)$ has no zeros in $G$. In this setting, we say that the sequence $\left(f_{n}\right)$ is associated sequence to the sequence $\left(x_{n}\right)$ and that $f_{0}$ is the associated limit.

Theorem 2.3. (a) Suppose that $h$ is a euclidean harmonic K-qc mapping from the unit ball $\mathbb{B} \subset \mathbb{R}^{n}$ onto a bounded convex domain $D=h(\mathbb{B})$, which contains the ball $B\left(h(0) ; R_{0}\right)$.

(i.7) If $h$ has Jacobian non zero normal family-property (or $h \in Q_{H}$ ), then $h$ is co-Lipschitz on $\mathbb{B}$.

(i.8) If $\log J_{h}$ is $q$-superharmonic, then $J_{h} \geq c$.

Proof. (i.7): Suppose that there is sequence $x_{n}$ such that $\left|h_{r}^{\prime}\left(x_{n}\right)\right| \rightarrow 0$ and set $d_{n}=d\left(x_{n}\right)$. Since by Theorems 2.1 and 2.2. $\underline{J}\left(x_{n}\right) \succeq \frac{d_{*}}{d} \succeq c$, there is a point $y_{n} \in \underline{B}\left(x_{n}\right)$ such that $\left|h_{r}^{\prime}\left(y_{n}\right)\right| \succeq c$. Apply a normal family argument on $h_{n}(x)=$ $\frac{1}{d_{* n}} h\left(\left(d_{n} x+x_{n}\right)\right)$.

(i.8) follows from Theorem 2.1(i.8), and Lemma 2.2

Using the Thom splitting lemma, we can prove

Proposition 2.1. Suppose that $f$ is real-valued function defined at a neighboorhood $U\left(x_{0}\right)$ of a point $x_{0} \in \mathbb{R}^{n}, f$ has partial derivatives up to the order 3 at $x_{0}$ and that $f: U\left(x_{0}\right) \rightarrow \mathbb{R}^{n}$ is injective, where $U\left(x_{0}\right)$ is a neighborhood of $x_{0}$ in $\mathbb{R}^{n}$. If $\partial_{k} f\left(x_{0}\right)=0$, then $\partial_{i j}^{2} f\left(x_{0}\right)=0, i, j=1,2, \cdots, n$, that is $\operatorname{Hess}(\mathrm{f})\left(\mathrm{x}_{0}\right)=[0]$. 
Frequently we use notation $X=(x, y, z) \in \mathbb{R}^{3}$. If we work in $\mathbb{R}^{n}$ it is convenient to switch the notation to $x=\left(x^{1}, x^{2}, \cdots, x^{n}\right) \in \mathbb{R}^{n}$.

Example 3. Let $a \neq 0$. A radial mapping $f_{a}$ in n-space is given by: $f(X)=$ $f_{a}(X)=|X|^{a-1} X$, where $X \in \mathbb{R}^{n}$. Prove

(i.2) $K_{I}(f)=|a|, K_{O}(f)=|a|^{n-1}$ if $|a| \geq 1$; in particular $K\left(f_{3}\right)=K_{O}\left(f_{3}\right)=3^{n-1}$; $K_{I}(f)=|a|^{1-n}, K_{O}(f)=|a|^{-1}$ if $|a| \leq \overline{1}$.

In particular, for $n=3, X=(x, y, z) \in \mathbb{R}^{3}$,

$K_{I}(f)=|a|, K_{O}(f)=|a|^{2}$ if $|a| \geq 1$;

Now we consider 3 -space.

(i.3) For $a=3$ set $g=f_{3}$; then $\partial_{k} g(0)=0, \partial_{i j}^{2} g(0)=0$ and $g^{1}(X)=x^{3}+x y^{2}+x z^{2}$.

(i.4) For $|a| \geq 1, f_{a}$ is co-Lipschitz on $\mathbb{B} \backslash F$, where $F$ is a compact subset of $\mathbb{B}$.

Set $x^{\prime}=f(x), x=\left(x^{1}, x^{2}, x^{3}\right)$; then $\left|x^{\prime}\right|=|x|^{a}$ and by the cosine formula, we find $|x|^{2 a}+|y|^{2 a}-\left|x^{\prime}-y^{\prime}\right|^{2}=|x|^{a-1}|y|^{a-1}\left(|x|^{2}+|y|^{2}-|x-y|^{2}\right)$.

$$
\left|x^{\prime}-y^{\prime}\right|^{2}=|x|^{a-1}|y|^{a-1}|x-y|^{2}+R(x, y) \text {, }
$$

where $R(x, y)=|x|^{2 a}+|y|^{2 a}-|x|^{a+1}|y|^{a-1}-|x|^{a-1}|y|^{a+1}$.

Without loss of generality we can suppose that $|y|=\lambda|x|, 0 \leq \lambda \leq 1$. Then $R(x, y)=\left(1-\lambda^{a-1}\right)\left(1-\lambda^{a+1}\right)|x|^{2 a} \geq 0$. Therefore $\left|x^{\prime}-y^{\prime}\right|^{2} \geq|x|^{a-1}|y|^{a-1}|x-y|^{2}$ and thus

$$
\left|x^{\prime}-y^{\prime}\right| \geq \lambda^{(a-1) / 2}|x|^{a-1}|x-y|
$$

We need the following Proposition concernig the distortion property of qr mappings.

Proposition $2.2([37])$. Let $f: \mathbb{B}^{n} \rightarrow \mathbb{B}^{n}$ be $K$-qr, $f(0)=0$ and $\alpha=K_{I}(f)^{1 /(1-n)}$. Then

(i.5) $|f(x)| \leq \varphi_{K, n}(|x|) \leq \lambda_{n}^{1-\alpha}|x|^{\alpha}$.

(i.6) If $g: \mathbb{B}^{n} \rightarrow \mathbb{B}^{n}$ is $K-q c, g(0)=0$ and $1 / \alpha=K_{I}\left(g^{-1}\right)^{1 /(n-1)}$, then $m|x|^{1 / \alpha} \leq|g(x)|$.

Suppose that $g$ is analytic (more generally $C^{(3)}$ at 0$), g(0)=0, \partial_{k} g(0)=0$ and $\partial_{i j}^{2} g(0)=0$. Then $\left|\partial_{k} g(x)\right| \leq M|x|^{2}$ and therefore $|g(x)-g(y)| \leq M|x|^{2}|x-y|$ if $|y| \leq|x|$. In particular, $|g(x)| \leq M|x|^{3}$.

Note that, if in addition, $f$ is $C^{(4)}$ at 0 and $\partial_{i j k}^{3} g(0)=0$, then $|g(x)-g(y)| \leq$ $M|x|^{3}|x-y|$ if $|y| \leq|x|$ and $f_{a}+g$ is K-qc for $a<4$. In particular, $|g(x)| \leq M|x|^{4}$.

Proposition 2.3. Suppose that $f$ has partial derivatives up to the order 3 at a point $x_{0} \in \mathbb{R}^{n}$ and that $f: U\left(x_{0}\right) \rightarrow \mathbb{R}^{n}$ is $K$-qc, where $U\left(x_{0}\right)$ is a neighborhood of $x_{0}$ in $\mathbb{R}^{n}$. If $\partial_{k} f\left(x_{0}\right)=0$ and $\partial_{i j}^{2} f\left(x_{0}\right)=0$, then $K_{O}(f) \geq 3^{n-1}$ on $U\left(x_{0}\right)$.

Example 3 shows that the result is optimal.

Proof. By the Taylor formula, there is $M$ such $|f(x)| \leq M|x|^{3}$.

If $1 / \alpha=K_{I}\left(g^{-1}\right)^{1 /(n-1)}$, then by Proposition 2.2. $m|x|^{1 / \alpha} \leq|g(x)| \leq M|x|^{3}$. Hence $K_{O}^{1 /(n-1)} \geq 3$, and therefore $K_{O} \geq 3^{n-1}$, where $K_{O}(g)=K_{I}\left(g^{-1}\right)$.

Proposition 2.4. Suppose that $f$ has continuous partial derivatives up to the order 3 at the origin 0 and that $f: U(0) \rightarrow \mathbb{R}^{n}$ is $K$-qc, where $U(0)$ is a neighborhood of 0 in $\mathbb{R}^{n}$. If $K_{O}(f)<3^{n-1}$, then $J(f, 0) \neq 0$.

In particular, if $g$ is analytic (more generally $C^{(3)}(U(0))$ or $g$ only has partial derivatives up to the order 3$)$, and if $g$ is K-qc with $K_{O}(g)<3^{n-1}$, then $J(g, 0) \neq 0$. 
Proof. Contrary suppose that $J(f, 0)=0$. Since $f$ is K-qc, $\partial_{k} f\left(x_{0}\right)=0$, hence by Proposition 2.1 we find $\partial_{i j}^{2} f\left(x_{0}\right)=0$. Now, by Proposition 2.3, $K_{O}(f) \geq 3^{n-1}$ and this yields a contradiction.

Theorem 2.4. If in addition to hypothesis (a) of Theorem 2.3 we suppose that $h$ is qc with $K_{O}(h)<3^{n-1}$, then $h$ is co-Lipschitz on $\mathbb{B}$.

Proof. The proof follows from Theorem 2.3 and Proposition 2.4. We leave the details to the interested reader.

\section{The Lower Bound of the Jacobian in $\mathbb{R}^{3}$}

It seems a natural project to generalize and develop the arguments used in planar theory of harmonic mappings to harmonic functions in domains of $\mathbb{R}^{n}, \geq 3$. In Section 1 we used the fact that every complex harmonic function $h$ on simple connected planar domain, can be written in the form $h=f+\bar{g}$, where $f$ and $g$ are holomorphic that $\left|f^{\prime}\right|$ satisfies minimum principle. There is no appropriate analogy of this result in space. The next example shows that the minimum principle does not hold for modulus of vector valued harmonic mapping.

Example 4. Define $h(M)=\left(x, y, x^{2}+y^{2}-1-2 z^{2}\right)$, where $M=(x, y, z), f_{0}(x, y)=$ $\left(x, y, x^{2}+y^{2}-1\right)$ and $G=\left\{(x, y, z): z<x^{2}+y^{2}-1\right\}$. Then $J(h)=-4 z$, the restriction of $h$ on xy-plane is $f_{0}, \Gamma_{f_{0}}=\partial G$ and $h\left(\mathbb{R}^{3}\right)=G$. Let $d(M)=|O M|$. Then $d$ attains minimum on $\Gamma_{f_{0}}$ at some point $M_{1}$ and there is a point $M_{0}=$ $\left(x_{0}, y_{0}, 0\right)$ such that $f_{0}\left(M_{0}\right)=M_{1}=h\left(M_{0}\right), h$ maps $\mathbb{R}^{3}$ onto $G$ and therefore $|h|$ attains minimum at $M_{0}$.

If $h$ is a harmonic mapping from a domain in $\mathbb{R}^{n}$ to $\mathbb{R}^{n}$, then $\left|h_{x_{k}}^{\prime}\right|$ is subharmonic, but it does not satisfy minimum principle in general (adapt the above example to the dimension $n \geq 3$ ).

In fact, Lewy's theorem is false in dimensions higer than two (see [7] p. 25-27 for Wood's counterexample).

Consider the polynomial map from $\mathbb{R}^{3}$ to $\mathbb{R}^{3}$ defined by $h(x, y, z)=(u, v, w)$, where

$$
u=x^{3}-3 x z^{2}+y z, \quad v=y-3 x z, \quad w=z .
$$

a calculation shows that $h$ has the Jacobian

$$
J_{h}(x, y, z)=3 x^{2}
$$

which vanishes on the plane $x=0$. Jacobian of $C^{1}$ orientation preserving mapping $f$ is nonnegative. Iwaniec [1] suggest a project (for students): Generalize and develop the arguments used in planar theory of harmonic mappings to gradient mappings of harmonic functions in domains of $\mathbb{R}^{3}$. Recall that, in planar theory of harmonic mappings we used

(I0) version of Hall and

(II.0) If analytic function does not vanish then its modulus satisfies minimum principle.

For harmonic gradient mapping in 3-space Proposition 3.2 and Theorem 3.1 are analogy of (I0) and (II.0) respectively.

For $n=3$, Lewy proved that the Hessian of a harmonic function (the determinant of its matrix of second derivatives) cannot vanish at an interior point of its 
domain without changing sign, unless it vanishes identically. More precisely, if the Hessian vanishes at some interior point $x_{0}$ without vanishing identically, then in each neighborhood of $x_{0}$ it must take both positive and negative values. But the Jacobian of a harmonic mapping $f=\operatorname{grad} u$ is the Hessian of $u$.

As a consequence, the Jacobian of a locally univalent harmonic gradient mapping from $\mathbb{R}^{3}$ to $\mathbb{R}^{3}$ cannot vanish at any interior point of its domain. Gleason and Wolff 10 generalized this result to $\mathbb{R}^{n}$.

Throughout this text the subscripts with variables $x, y$ and $z$ designate partial derivatives.

A vector field $F=\left(f^{1}, f^{2}, f^{3}\right)$ is said to satisfy the Cauchy-Riemann equations (CR-equations, for short) if its coordinates, (conjugate harmonic functions) satisfy: $f_{y}^{1}=f_{x}^{2}, f_{z}^{1}=f_{x}^{3}, f_{z}^{2}=f_{y}^{3}, f_{x}^{1}+f_{y}^{2}+f_{z}^{3}=0$, locally $F=\operatorname{grad} \phi$ and $\Delta \phi=0$.

Equivalently, the Jacobian matrix of $F$ is symmetric and has trace 0.

Theorem 3.1 (Lewy-Gleason-Wolff, 10]). Logarithm of modulus of the Hessian of a harmonic function in a domain $\Omega \subset \mathbb{R}^{3}$ is superharmonic outside its zeros. Precisely, $\Delta \ln |H| \leq 0$, wherever $H \neq 0$.

Remark. This inequality fails in dimensions greater than 3. Obviously, it holds (as equality) for planar harmonic functions.

Proposition 3.1. Suppose Hessian determinant $H$ of a harmonic function in a domain $\Omega \subset \mathbb{R}^{3}$ is positive. Then for every compact $F \subset \Omega$ we have $\inf _{F} H \geq$ inf $f_{\partial F} H$.

In particular, if $f$ is injective harmonic gradient mapping, we have $\inf _{F} J(f) \geq$ inf $f_{\partial F} J(f)$.

Using a normal family argument one can prove (see subsection 3.1 for details):

Proposition 3.2. 28 Suppose that $G$ and $G^{\prime}$ are domains in $\mathbb{R}^{3}:$ If $f: G \rightarrow G^{\prime}$ is injective harmonic K-qc gradient mapping, then $f$ has weak $H$-property.

A more general result will appear in a forthcoming paper.

Proposition 3.3. Suppose that

(a1): $f$ is injective harmonic gradient mapping from $\mathbb{B}$ onto $D \subset \mathbb{R}^{3}$ and

(b1): partial derivatives of $f$ have continuous extension to $\overline{\mathbb{B}}$

(i.1) If $J_{h} \geq j_{0}>0$ on $\mathbb{S}^{2}$, then $J_{h} \geq j_{0}$ on $\overline{\mathbb{B}}$.

(i.2) If in addition $f$ is $K$-qc, then $f: \mathbb{B} \rightarrow D$ is bi-Lipschitz.

Theorem 3.2. Suppose that

(a1) $f$ is continuous on $\overline{\mathbb{B}}$, and univalent harmonic K-qc gradient mapping from $\mathbb{B}$ onto convex domain $D \subset \mathbb{R}^{3}$ and

(b1) partial derivatives of $f$ have continuous extension to $\overline{\mathbb{B}}$.

(ii.1) Then $f: \mathbb{B} \rightarrow D$ is bi-Lipschitz and in particular $f^{-1}: D \rightarrow \mathbb{B}$ is $L$ Lipschitz.

(ii.2) If $f$ is injective harmonic $K$-qc gradient mapping from $\mathbb{B}$ onto convex bounded domain $D \subset \mathbb{R}^{3}$, then $f$ is co-Lipschitz.

Proof. (i.1), (i.2) and (ii.1) are corollary of Proposition 3.1

(ii.2) is a corollary of Proposition 3.2 and Theorem 2.1 (i.3).

Note that the above outline of proof of (ii.2) is not based on Theorem 3.1 (see subsection 3.1 for more details). Astala-Manojlović first made publicly available proof of (ii.2) in Math.Arxiv, [5]. 
In particular, (ii.2) yields:

Proposition 3.4. (b) Suppose that $f$ is univalent harmonic gradient mapping from $\mathbb{B}^{3}$ onto itself. Then $f$ is co-Lipschitz.

In communication between V. Zorich and the author, the question was asked to find examples of functions that satisfy the condition (c). For example, if $u=$ $x^{2}+y^{2}-2 z^{2}$, then $f=\nabla u=(2 x, 2 y,-4 z)$ is injective harmonic gradient mapping from $\mathbb{B}^{3}$ onto the ellipsoid.

If $u$ is real-valued function such that $f=\nabla u=(x, y, z)$, then $u=x^{2} / 2+y^{2} / 2+$ $z^{2} / 2+c$.

In particular, $I d$ is not harmonic gradient mapping.

In complex plane, if $u$ is real-valued harmonic function, then $u_{z}=\frac{1}{2}\left(u_{x}^{\prime}-u_{y}^{\prime}\right)$ is analytic function and therefore $\nabla u=\bar{F}$, where $F=2 u_{z}$ is analytic function.

3.1. Hall lemma and co-Lipschitz property of qc gradient harmonic mappings. Here, we outline a proof of Theorem 3.2, the part (ii.2), stated here as:

(A0) A euclidean gradient harmonic mapping from the unit ball $\mathbb{B} \subset \mathbb{R}^{3}$ onto a bounded convex domain is co-Lipschitz.

Note that our proof of (A0) is based on the Hall lemma and a normal family argument and our approach is different from that in [5; see also 6 .

We first prove Hall lemma for harmonic injective mappings in $\mathrm{n}$-dimensional space.

If $G$ and $G^{\prime}$ are domains in $\mathbb{R}^{n}$, by $Q C H\left(G, G^{\prime}\right)$ (respectively $Q C H_{K}\left(G, G^{\prime}\right)$ ) we denote the set of Euclidean harmonic quasiconformal mappings (respectively K-qc) of $G$ onto $G^{\prime}$.

Lemma 3.1. Let $F_{K}$ be a family of harmonic K-qc mapping $f: \mathbb{B} \rightarrow \mathbb{R}^{n}$ such that for $f \in F_{K}$, $J(f)$ has no zeros, $F_{K}$ is closed with respect to uniform convergence, $f(\mathbb{B}) \supset \mathbb{B}$ and $f(0)=0$. Then there is a constant $c>0$ such that if $f \in F_{K}$ is harmonic K-qc mapping $f(\mathbb{B}) \supset \mathbb{B}, f(0)=0$, then $\Lambda_{f}(0) \geq c$.

Proof. Suppose, on the contrary, that there is a sequence $f_{n} \in F_{K}$ such that $\Lambda_{f_{n}}(0) \rightarrow 0$. The sequence $\left(f_{n}\right)$ forms a normal family and there is a subsequence of $f_{n}$ which converges uniformly to a limit $f_{0} \in F_{K}$; this is a contradiction.

Our further considerations are related to weak $H$-property (see Definition 2.2).

By Hall lemma planar euclidean harmonic mappings have $H$-property. Let $Q C H_{K}^{0}=Q C H_{K}^{0}(\mathbb{B}, D)$ be family of gradient harmonic mappings which maps $\mathbb{B}$ onto $D$.

Definition 3.1. We say that $\underline{F} \subset Q C H_{K}\left(G, G^{\prime}\right)$ has $J$-property if $F$ is closed with respect to uniform convergence, and for $f \in \underline{F}, J(f)$ has no zeros in $G$.

Now we sketch a proof of the statement (A0) in few steps (A1-A5):

(A1) If $\underline{F} \subset Q C H_{K}\left(G, G^{\prime}\right)$ has $J$-property, then $f \in \underline{F}$ has weak $H$-property.

Outline of proof. By Lemma 3.1, there is a constant $c>0$ such that $d(x) \Lambda_{f}(x) \geq$ $c d(f x), x \in G$.

(A2) Suppose that $h$ is a euclidean harmonic mapping from the unit ball $\mathbb{B} \subset \mathbb{R}^{n}$ onto a bounded convex domain $D=h(\mathbb{B})$. Then there is a constant $c>0$ such that $d^{*}(f(x)) \geq c d(x), x \in \mathbb{B}$.

(A3) If $f$ is injective harmonic gradient mapping, which maps $\mathbb{B} \subset \mathbb{R}^{n}$ onto a bounded convex domain $D=h(\mathbb{B})$, then any associated limit of $f$ is harmonic 
gradient mapping.

In dimension $n=3$, then it has Jacobian non zero normal family-property.

(A4) If $\underline{F} \subset Q C H_{K}(B, D)$ has $H$-property, then every $f \in \underline{F}$ is co-Lipschitz.

By (A1) there is a constant $c>0$ such that $d(x) \Lambda_{f}(x) \geq c d^{*}(f(x)), x \in \mathbb{B}$, and by (A2), a constant $c_{1}>0$ such that $d^{*}(f(x)) \geq c_{1} d(x)$. Hence $\lambda_{f}(x) \geq c_{2}$, where $c_{2}=c c_{1} / K$.

(A5) In 3-dimensional space, $Q C H_{K}^{0}=Q C H_{K}^{0}(\mathbb{B}, D)$ has $H$-property.

From (A1-A5), it follows (A0).

\section{Appendix 1}

In this review section we follow [25, 26. First we recall some results from Section 1 (Theorem 1.2) and prove (I0) version of Hall lemma.

Theorem 4.1. Let $h$ be an euclidean harmonic orientation preserving univalent mapping of the unit disc onto convex domain $\Omega$. If $\Omega$ contains a disc $B(a ; R)$ and $h(0)=a$ then

$$
|\partial h(z)| \geq \frac{R}{4}, z \in \mathbb{D}
$$

As a corollary of the previous Theorem we obtain

Theorem 4.2. Let $h$ be an euclidean harmonic orientation preserving $K$-qc mapping of the unit disc onto convex domain $\Omega$. If $\Omega$ contains a disc $B(a ; R)$ and $h(0)=a$ then

$$
\begin{gathered}
|\partial h(z)| \geq \frac{R}{4}, z \in \mathbb{D}, \\
l_{h}(z) \geq \frac{1-k}{4} R .
\end{gathered}
$$

Let $c=\frac{1-k}{4} R$. Since

$$
|\bar{D} h(z)| \leq k|D h(z)|,
$$

it follows that $l_{h}(z) \geq c=\frac{1-k}{4} R$ and therefore $\left|h\left(z_{2}\right)-h\left(z_{1}\right)\right| \geq c\left|z_{2}-z_{1}\right|$.

As a corollary of Theorem 4.1 we obtain

Proposition 4.1. Let $h$ be an euclidean harmonic orientation preserving univalent mapping of the unit disc into $\mathbb{C}$ such that $f(\mathbb{D})$ contains a disc $B_{R}=B(a ; R)$ and $h(0)=a$. Then

$$
|\partial h(0)| \geq \frac{R}{4}
$$

Proof: Let $V=V_{R}=h^{-1}\left(B_{R}\right)$ and $\varphi$ be a conformal mapping of the unit disc $U$ onto $V$ such that $\varphi(0)=0$ and let $h_{R}=h \circ \varphi$. By Schwarz lemma

$$
\left|\varphi^{\prime}(0)\right| \leq 1 \text {. }
$$

Since $\partial h_{R}(0)=\partial h(0) \varphi^{\prime}(0)$, by Proposition 4.1 we get $\left|\partial h_{R}(0)\right|=|\partial h(0)|\left|\varphi^{\prime}(0)\right| \geq$ $\frac{R}{4}$. Hence, using $(0.2)$ we get $(0.1)$.

Also as an immediate corollary of Theorem 4.1 we obtain 
Theorem 4.3 (16, 18]). Let $h$ be an euclidean harmonic diffeomorphism of the unit disc onto convex domain $\Omega$. If $\Omega$ contains a disc $B(a ; R)$ and $h(0)=a$ then

$$
D(h)(z) \geq \frac{1}{16} R^{2}, z \in \mathbb{D},
$$

where $D(h)(z)=|\partial h(z)|^{2}+|\bar{\partial} h(z)|^{2}$.

The following example shows that previous results are not true if we omit the condition $h(0)=a$.

Example. The mapping

$$
\varphi_{b}(z)=\frac{z-b}{1-\bar{b} z},|b|<1
$$

is a conformal automorphism of the unit disc onto itself and

$$
\left|\varphi_{b}^{\prime}(z)\right|=\frac{1-|b|^{2}}{|1-\bar{b} z|^{2}}, z \in \mathbb{D} .
$$

In particular $\varphi_{b}^{\prime}(0)=1-|b|^{2}$.

Heinz proved (see [13]) that if $h$ is a harmonic diffeomorphism of the unit disc onto itself such that $h(0)=0$, then

$$
D(h)(z) \geq \frac{1}{\pi^{2}}, z \in \mathbb{D} .
$$

Using Proposition 4.1 we can prove Heinz theorem:

Theorem 4.4 (Heinz). There exists no euclidean harmonic diffeomorphism from the unit disc $\mathbb{D}$ onto $\mathbb{C}$.

Note that this result was a key step in his proof of the Bernstein theorem for minimal surfaces in $\mathbb{R}^{3}$.

Schoen obtained a nonlinear generalization of Proposition 4.1 by replacing the target by complete surface of nonnegative curvature (see Proposition 2.4 [38]) and using this result he proved

Theorem 4.5 (Schoen). There exists no harmonic diffeomorphism from the unit disc onto a complete surface $(S, \rho)$ of nonnegative curvature $K_{\rho} \geq 0$.

Suppose $f$ is a harmonic diffeomorphism from $B_{r}$ to $(S, \rho)$ and $\operatorname{dist}\left(f(0), \partial\left(f\left(B_{r}\right)\right) \geq\right.$ $R$. Then it suffices to show that

$$
|d f|^{2}(0) \geq C \frac{R^{2}}{r^{2}}
$$

where $C$ is a universal constant. By hypothesis, we have $|\partial f|>|\bar{\partial} f| \geq 0$ and

$$
\Delta l n|\partial f|=-K_{\rho} J_{f} \leq 0 .
$$

If we define a Riemannian metric $\lambda$ on $B_{r}$ by $\lambda=|\partial f|^{2}|d z|^{2}$, then (4.3) implies $K_{\lambda} \geq 0$. Therefore $\operatorname{dist}\left(0, \partial\left(B_{r}\right) \geq \frac{1}{2} \operatorname{dist}\left(f(0), \partial\left(f\left(B_{r}\right)\right) \geq \frac{1}{2} R\right.\right.$

Lemma 4.1. If $\sigma$ is a metric density of nonnegative curvature $K_{\sigma} \geq 0$ on $B_{r}$ and $d=\operatorname{dist}_{\sigma}\left(0, T_{r}\right)$, then $\sigma(0) \geq C \frac{d^{2}}{r^{2}}$, where $C$ is a universal constant. 
A proof can be given by means the estimate of harmonic function in terms of curvature (Cheng-Yau, CPAM 28, 333-354 (1975)). We apply this lemma to metric density $\lambda=|\partial f|^{2}$. By the above estimate,

$$
|\partial f|^{2}(0) \geq C \frac{R^{2}}{r^{2}}
$$

This proves the theorem.

Question 2. Can we prove Lemma 4.1 elementary? Note that $\ln \sigma$ is superharmonic function. Therefore $\ln \frac{1}{\sigma}$ and $\frac{1}{\sigma}$ are subharmonic functions.

4.1. Distortion of conformal mappings. The following form of Koebe's OneQuarter Theorem applies in fact to all conformal mappings.

Theorem 4.6. Suppose that $f$ is bijective conformal in $D$ and $f(D)=D^{\prime}, z_{0} \in D$. Then

$$
\frac{1}{4}\left|f^{\prime}\left(z_{0}\right)\right| \operatorname{dist}\left(z_{0}, \partial D\right) \leq \operatorname{dist}\left(f\left(z_{0}\right), \partial D^{\prime}\right) \leq 4\left|f^{\prime}\left(z_{0}\right)\right| \operatorname{dist}\left(z_{0}, \partial D\right) .
$$

If we set $d=d\left(z_{0}\right)=\operatorname{dist}\left(z_{0}, \partial D\right)$ and $d^{\prime}=d^{\prime}\left(f\left(z_{0}\right)=\operatorname{dist}\left(f\left(z_{0}\right), \partial D^{\prime}\right)\right.$ then (iv.1): $d^{\prime} \asymp d$.

Proof. Let $d=d\left(z_{0}\right)=d_{D}\left(z_{0}\right)=\operatorname{dist}\left(z_{0}, \partial D\right), d^{\prime}=d\left(f\left(z_{0}\right)\right)=d_{D^{\prime}}\left(f\left(z_{0}\right)\right)=$ $\operatorname{dist}\left(f\left(z_{0}\right), \partial D^{\prime}\right)$;

$$
g(z)=\frac{f(z)-f\left(z_{0}\right)}{d \cdot f^{\prime}\left(z_{0}\right)} \text { and } f_{0}(z)=g\left(z_{0}+z d\right) .
$$

Set $D_{0}=g\left(\mathbb{D}\left(z_{0} ; d\right)\right)$ and $d_{0}=\operatorname{dist}\left(g\left(z_{0}\right), \partial D_{0}\right)$. Note that $d_{1}=\operatorname{dist}\left(g\left(z_{0}\right), \partial D\right)=$ $\frac{d^{\prime}}{d\left|f^{\prime}\left(z_{0}\right)\right|}$ and $d_{1} \geq d_{0}$. Since $f_{0}^{\prime}(0)=1$, it follows from Koebe's One-Quarter Theorem, applied to $f_{0}$ that $d_{0} \geq 1 / 4$. Hence, since $\frac{d^{\prime}}{d\left|f^{\prime}\left(z_{0}\right)\right|}=d_{1} \geq d_{0} \geq 1 / 4$, we get the left inequality.

Koebe's Theorem applied to $f^{-1}$ at $w_{0}=f\left(z_{0}\right)$ gives $\frac{1}{4}\left|\left(f^{-1}\right)^{\prime}\left(w_{0}\right)\right| d^{\prime} \leq d$ and the right inequality follows.

If we define $D_{f}^{*}\left(z_{0}\right)=\frac{d\left|f^{\prime}\left(z_{0}\right)\right|}{d^{\prime}}$, we can reformulate the above theorem as $1 / 4 \leq$ $D_{f}^{*}\left(z_{0}\right) \leq 4$. The interested reader can check that

$$
D_{f^{-1}}^{*}\left(w_{0}\right)=D_{f}^{*}\left(z_{0}\right) .
$$

The following two basic theorems are important for our research.

Theorem 4.7 (Kellogg, see for example [9, 8]). If a domain $D=\operatorname{Int}(\Gamma)$ is $C^{1, \alpha}$, $0<\alpha<1$, and $\omega$ is a conformal mapping of $\mathbb{D}$ onto $D$, then $\omega^{\prime}$ and $\ln \omega^{\prime}$ are in $\operatorname{Lip}_{\alpha}$. In particular, $\left|\omega^{\prime}\right|$ is bounded from above and below on $\mathbb{D}$.

Theorem 4.8 (Kellogg and Warschawski, see 41], Theorem 3.6). If a domain $D=\operatorname{Int}(\Gamma)$ is $C^{2, \alpha}$ and $\omega$ is a conformal mapping of $\mathbb{D}$ onto $D$, then $\left|\omega^{\prime \prime}\right|$ has a continuous extension to the boundary. In particular it is bounded from above on $\mathbb{D}$. 
4.2. The uniformization theorem. The uniformization theorem says that every simply connected Riemann surface is conformally equivalent to one of the three domains: the open unit disk, the complex plane, or the Riemann sphere. In particular it admits a Riemannian metric of constant curvature. This classifies Riemannian surfaces as elliptic (positively curved rather, admitting a constant positively curved metric), parabolic (flat), and hyperbolic (negatively curved) according to their universal cover.

The uniformization theorem is a generalization of the Riemann mapping theorem from proper simply connected open subsets of the plane to arbitrary simply connected Riemann surfaces. The uniformization theorem implies a similar result for arbitrary connected second countable surfaces: they can be given Riemannian metrics of constant curvature. Every Riemann surface is the quotient of a free, proper and holomorphic action of a discrete group on its universal covering and this universal covering is holomorphically isomorphic (one also says: "conformally equivalent") to one of the following: the Riemann sphere, the complex plane or the unit disk in the complex plane. Koebe proved the general uniformization theorem that if a Riemann surface is homeomorphic to an open subset of the complex sphere (or equivalently if every Jordan curve separates it), then it is conformally equivalent to an open subset of the complex sphere. In 3 dimensions, there are 8 geometries, called the eight Thurston geometries. Not every 3-manifold admits a geometry, but Thurston's geometrization conjecture proved by Grigori Perelman states that every 3 -manifold can be cut into pieces that are geometrizable. The simultaneous uniformization theorem of Lipman Bers shows that it is possible to simultaneously uniformize two compact Riemann surfaces of the same genus $>1$ with the same quasi-Fuchsian group. The measurable Riemann mapping theorem shows more generally that the map to an open subset of the complex sphere in the uniformization theorem can be chosen to be a quasiconformal map with any given bounded measurable Beltrami coefficient.

\section{Appendix 2, Harmonic Maps Between surfaces}

In 14 it is given self-contained account of the results on harmonic maps between surfaces. This treatment contains several simplifications and unifications compared to the presentations available in the existing literature. Upper and lower bounds for the sectional curvature $K$ of a manifold are often denoted by $k^{2}$ and $-\omega^{2}$, i.e. $-\omega^{2} \leq K \leq k^{2}$. This notation avoids square roots. It differs, however, from the terminology in some of the papers frequently referred to in the book [14.

Here we give short review of results related to our consideration in section 1 . First the lower bound for Jacobinan are considerd.

Theorem 5.1. Suppose $u: \mathbb{D} \rightarrow \Sigma$ is harmonic, and $u(D) \subset B(p, M)$, where $B(p, M)$ again is a disc with radius $M<\pi / 2 k$. Suppose that $\partial u(\mathbb{D})=u(\mathbb{T})$ and that $g:=u \mid T: T \rightarrow \partial u(D)$ is a $C^{2}$ - diffeomorphism with

(d1) $0<b \leq\left|g^{\prime}(t)\right|$ for all $t \in[0,2 \pi]$.

Assume furthermore that $g(\mathbb{T})$ is strictly convex w.r.t, $u(\mathbb{D})$, and that we have the following estimates for the geodesic curvature of $g(\mathbb{T})$

(d2) $0<a_{1} \leq \kappa_{g}(\underline{g}(t)) \leq a_{2}$ for all $t \in[0,2 \pi]$.

Then (iv.1):

$J \geq \delta_{1}^{-1}$, where $\delta_{1}=\delta_{1}\left(\omega, k, M, \tau, a_{1}, a_{2}, b,|g|_{1, \alpha}\right)$ ( $\tau$ is given in Thm. 6.2 [14]). 
Note that it is not assumed in the theorem that $u$ is univalent; we needed only that $u$ maps $\mathbb{D}$ onto the convex side of $u(\mathbb{T})$. If $u$ is an injective harmonic map, then $J \geq \delta_{2}^{-1}$ on $\mathbb{D}$.

Proposition 5.1. Assume $u: \mathbb{D} \rightarrow \Sigma$ is an injective harmonic map, where $u(D) \subset$ $B(p, M)$, and $B(p, M)$ is a disc with radius $M<\pi / 2 k$. Suppose that $g:=u \mid \mathbb{T} \in$ $C^{1, \alpha}$, and that $(\mathrm{d} 1)$ and $(\mathrm{d} 2)$ hold. Then (iii.2): for all $z \in \mathbb{D}, J \geq \delta_{2}^{-1}$, where $\delta_{2}=\delta_{2}\left(\omega, k, M, \tau, a_{1}, a_{2}, b,|g|_{1, \alpha}\right)$.

We define $d_{*}(q):=-\operatorname{dist}(q, \partial u(D))$ for $q \in u(D)$. Since $\triangle\left(d_{*} \circ u\right) \geq a_{1} b^{2}, \quad d_{*} \circ u$ is subharmonic function. This will enable us to get a lower bound for the radial derivative of $d_{*} \circ u$ at boundary points with the argument of the boundary lemma of E. Hopf.

Taking Cor. 6.2[14] into account, we can therefore find a neighborhood $V_{0}$ of $\mathbb{T}$ in $\mathbb{D}$ with the property that $d_{*}$ is a $C^{2}$ function with strictly convex level curves on $u\left(V_{0}\right)$. Suppose $z_{0} \in \mathbb{T}$; we can choose some $\operatorname{disc} B_{1}=B\left(z_{1}, r_{1}\right) \subset D, z_{0} \in S\left(z_{1}, r_{1}\right)$, in such a way that $\triangle\left(d_{*} \circ u\right) \geq a_{1} b^{2} / 2$ for $z \in B_{1}$.

Defining the auxiliary function $v$ via $v(z)=\frac{r_{1}^{2}}{8} a_{1} b^{2}\left(1-\frac{\left|z-z_{1}\right|^{2}}{r_{1}^{2}}\right)$, we find $\triangle v=$ $-a_{1} b^{2} / 2$ and therefore $\triangle\left(d_{*} \circ u+v\right) \geq 0$. The maximum principle now controls the derivative of $d_{*} \circ u+v$ at $z_{0}$ in the direction of the outer normal.

Now, using the above estimates, the existence of harmonic diffeomorphisms which solve a Dirichlet problem is considered.

Theorem 5.2. Suppose (e1): $\Omega$ is a compact domain with Lipschitz boundary $\partial \Omega$ on some surface, and that $\Sigma$ is another surface. We assume (e2): that $f: \bar{\Omega} \rightarrow \Sigma$ maps $\bar{\Omega}$ homeomorphically onto its image, that $f(\partial \Omega)$ is contained in some disc $B(p, M)$ with radius $M<\pi / 2 k$ (where $k^{2} \geq 0$ is an upper curvature bound on $B(p, M))$ and that the curves $f(\partial \Omega)$ are of Lipschitz class and convex w.r.t. $f(\Omega)$. Then (v.1): there exists a harmonic mapping $u: \Omega \rightarrow B(p, M)$ with the boundary values prescribed by $f$ which is a homeomorphism between $\bar{\Omega}$ and its image, and a diffeomorphism in the interior.

(v.2) Moreover, if $f \mid \partial \Omega$ is even a $C^{2}$-diffeomorphism between $C^{2}$-curves, then $u$ is a diffeomorphism up to the boundary.

First of all, $\partial \Omega$ is connected. Otherwise, $f(\partial \Omega)$ would consist of at least two curves, both of them convex w.r.t. $f(\Omega)$. Since $\Omega$ is homeomorphic to $f(\Omega)$, we conclude that $\Omega$ is a disc, topologically. Since there is a conformal map $\phi: D \rightarrow$ $g(\mathbb{D})$, one have to prove the theorem only for the case where $\Omega$ is the plane unit $\operatorname{disc} \mathbb{D}$.

We first assume that $f: \mathbb{T} \rightarrow f(\mathbb{T})$ is $C^{2}$-diffeomorphism between curves of class $C^{2, \alpha}$, that $f(\mathbb{T})$ is not only convex, but strictly convex, and that we have the following quantitative bounds

(e2) $\left|\underline{f}^{\prime}(t)\right| \geq b_{2}^{-1}$ and $\left|\underline{f}^{\prime \prime}(t)\right| \leq b_{1}$.

Now let $\Gamma$ be the parametrization of the boundary curve of $g(D)$ by arclength. If $l$ is length of $\partial g(D)$, then $\Gamma$ maps $[0, l]$ on $\partial g(D)$. We set $w(z, \lambda)=\lambda \Gamma^{-1}(\phi(z)+$ $(1-\lambda) \Gamma^{-1}(g(z)), \omega(z, \lambda)=\Gamma(w(t, \lambda)), z \in T, \lambda \in[0,1]$, and $\underline{\omega}(t, \lambda) \omega\left(e^{i t}, \lambda\right)$. Using (e2), one can check that

$$
\underline{\omega}(t, \lambda), \frac{\partial \underline{\omega}(t, \lambda)}{\partial t} \text { and } \frac{\partial^{2} \underline{\omega}(t, \lambda)}{\partial^{2} t} \text { are continuous functions of } \lambda \text {. }
$$

Let now $u_{\lambda}$ denote the harmonic map from $D$ to $B(p, M)$ with boundary values $\omega(\cdot, \lambda)$. In particular, $m(\lambda):=\inf _{z \in D}\left|J\left(u_{\lambda}\right)(z)\right|$ depends continuously on $\lambda$. We 
define $L:=\{\lambda \in[0,1]: m(\lambda)>0\} .0 \in L ;\left(u_{0}\right.$ is the conformal map $\left.\phi\right)$, and therefore $L$ is not empty.

By Proposition [5.1, $m(\lambda) \geq m_{0}>0$ for $\lambda \in L$. Since $m(\lambda)$ depends continuously on $\lambda$, (8.1.7) implies $L=[0,1]$. Thus, $u_{1}$ is a local diffeomorphism and a diffeomorphism between the boundaries of $D$ and $u_{1}(D)$, and consequently a global diffeomorphism by topology. Theorem 5.2 and the uniqueness theorem of Jager and Kaul (cf. Theorem 5.1 [14]) imply

Corollary 3. Under the assumptions of Thm. 5.2, each harmonic map which solves the Dirichlet problem defined by $g$ and which maps $\Omega$ into a geodesic disc $B(p, M)$ with radius $M<\pi / 2 k$, is a diffeomorphism in $\Omega$.

The above, we have assumed that the boundary of the image is strictly convex, and, in addition, that the boundary values are a diffeomorphism of class $C^{2}$. The theorem also holds for the case that

(f1): the boundary is only supposed to be convex and that

(f2): the boundary values are only supposed to induce a homeomorphism of the boundaries.

The procedure to handle the case (f1) is called the first approximation argument. It is a modification of the corresponding one given by E. Heinz.

The case (f1) of a general boundary is handled by an approximation by smooth curves. More precisely it is supposed that the boundary of the image $f(\mathbb{D})$ is only convex, while the boundary values $f$ are still assumed to be a diffeomorphism of class $C^{2}$. For approximation arguments in planar case see also [20, 11, 12].

Acknowledgement. I would like to thank V. Bozin and D. Kalaj for stimulating discussions related to this topic. In particular, D. Kalaj turned my attention on important consideration in [14] and informed me about Iwaniec -Onninen paper [12.

\section{REFERENCES}

[1] A. Abaob, M. Arsenović, M. Mateljević and A. Shkheam, Moduli of continuity of harmonic quasiregular mappings on bounded domains, Ann. Acad. Sci. Fenn. Math. 38(2013), 839-847.

[2] G. Alessandrini, V. Nesi: Invertible harmonic mappings, beyond Kneser, Ann. Scuola Norm. Sup. Pisa, Cl. Sci. (5) VIII (2009), 451-468.

[3] M. Arsenović, V. Kojić and M. Mateljevć, On Lipschitz continuity of harmonic quasiregular maps on the unit ball in $R^{n}$, Ann. Acad. Sci. Fenn. vol. 33, 2008, 315-318.

[4] K. Astala, F. W. Gehring, Quasiconformal analogues of theorems of Koebe and HardyLittlewood, Mich. Math. J. 32 (1985) 99-107.

[5] K. Astala, V. Manojlović, Pavlović's theorem in space, arXiv:1410.7575 [math.CV]

[6] V. Božin, M. Mateljevć, Bounds for Jacobian of harmonic injective mappings in n-dimensional space, arXiv:1502.00457 [math.AP]

[7] P. Duren, Harmonic mappings in the plane, Cambridge Univ. Press, 2004.

[8] G. M. Goluzin: Geometric function theory, Nauka, Moskva, 1966. (in Russian).

[9] G. M. Goluzin: Geometric theory of functions of a complex variable. Translations of Mathematical Monographs, Vol. 26 American Mathematical Society, Providence, R.I. 1969 vi+676 pp.

[10] S. Gleason, T. Wolff, Lewy's harmonic gradient maps in higher dimensions, Communications in Partial Differential Equations Volume 16, Issue 12, 1925-1968, 1991.

[11] T. Iwaniec, A path from Riemann's Conformal Mapping Theorem to Harmonic Homeomorphisms of Rado-Kneser-Choquet, (Helsinki \& Syracuse) Seminar Talk in AALTO, January 28, 2014.

[12] T. Iwaniec and J. Onninen, Radó-Kneser-Choquet theorem, Bull. London Math. Soc. 46 (2014) 12831291, http://blms.oxfordjournals.org/content/46/6/1283.full.pdf+html 
[13] E. Heinz, On one-to-one harmonic mappings, Pac. J. Math. 9 (1959), 101-105.

[14] J. Jost, Harmonic Maps Between surfaces, Springer-Verlag Berlin Heidel berg New York Tokyo, 1984.

[15] J. Jost, Two-dimensional Geometric Variational Problems, John Wiley \& Sons, 1991.

[16] D. Kalaj, Harmonic functions and quasiconformal mappings, in Serbian: Harmonijske funkcije i kvazikonformna preslikavanja, Master thesis 1998.

[17] D. Kalaj, Harmonic and quasiconformal functions between convex domains, Doctoral Thesis, Beograd, submited 2000, (2002).

[18] D. Kalaj, On harmonic diffeomorphisms of the unit disc onto a convex domain, Complex Variables, Theory Appl. 48, No. 2, 175-187 (2003).

[19] D. Kalaj, Quasiconformal harmonic functions between convex domains, Publ. Inst. Math., Nouv. Ser. 76(90) (2004). 3-20.

[20] D. Kalaj, Invertible harmonic mappings beyond Kneser theorem and quasiconformal harmonic mappings, Studia mathematica (ISSN:0039-3223), Volume 207, Issue 2, Dec. 2011, Page(s) $117-136$.

[21] D. Kalaj, A priori estimate of gradient of a solution to certain differential inequality and quasiconformal mappings, Journal d'Analyse Math. Volume 119, 2013, pp 63-88.

[22] D. Kalaj, M. S. Mateljević, Harmonic quasiconformal self-mappings and Möbius transformations of the unit ball. Pacific journal of mathematics. ISSN 0030-8730. $247: 2$ (2010) 389-406.

[23] D. Kalaj, M. Marković, M. Mateljević, Carathédory and Smirnov type theorems for harmonic mappings of the unit disk onto surfaces, Annales Academiae Scientiarum Fennicae Mathematica Volumen 38, 2013, 565-580.

[24] O. Martio, On harmonic quasiconformal mappings, Ann. Acad. Sci. Fenn., A 1, 425, 3-10 (1968)

[25] M. Mateljević, Estimates for the modulus of the derivatives of harmonic univalent mappings, Proceedings of International Conference on Complex Analysis and Related Topics (IX ${ }^{\text {th }}$ Romanian-Finnish Seminar, 2001), Rev Roum Math Pures Appliq (Romanian Journal of Pure and Applied mathematics) 47 (2002) 5-6, 709 -711.

[26] M. Mateljević: Distortion of harmonic functions and harmonic quasiconformal quasiisometry, Revue Roum. Math. Pures Appl. Vol. 51 (2006) 5-6, 711-722.

[27] M. Mateljević: Quasiconformality of harmonic mappings between Jordan domains 2, Filomat $26: 3$ (2012) 479-509.

[28] M. Mateljević, (a) Communications at Analysis Seminar, University of Belgrade,

(b) Lipschitz-type spaces, Quasiconformal and Quasiregular harmonic mappings and Applications, unpublished manuscript, 2008.

[29] M. Mateljević and M. Vuorinen, On harmonic quasiconformal quasi-isometries, J. Inequal. Appl., Volume 2010, Article ID 178732, 19 pages doi:10. 1155/2010/1787.

[30] M. Mateljević, Topics in Conformal, Quasiconformal and Harmonic maps, Zavod za udžbenike, Beograd 2012.

[31] M. Mateljević: Distortion of quasiregular mappings and equivalent norms on Lipschitztype spaces, Abstract and Applied Analysis Volume 2014 (2014), Article ID 895074, 20 pages http://dx.doi.org/10.1155/2014/895074 http://www.hindawi.com/journals/aaa/2014/895074/

[32] M. Mateljević, The lower bound for the modulus of the derivatives and Jacobian of harmonic univalent mappings http://arxiv.org/pdf/1501.03197v1.pdf] [math.CV] 13 Jan 2015

[33] G. Martin, The Theory of Quasiconformal Mappings in Higher Dimensions, I, arXiv:1311.0899 1 [math.CV] 4 Nov 2013

[34] M. Pavlović, Boundary correspondence under harmonic quasiconformal homeomorfisms of the unit disc, Ann. Acad. Sci. Fenn., Vol 27, (2002) 365-372.

[35] L. Tam, T. Wan, On quasiconformal harmonic maps, Pacific J Math, V 182 (1998), 359-383.

[36] J. Väisälä, Lectures on n-Dimensional Quasiconformal Mappings, Springer-Verlag, 1971.

[37] M. Vuorinen, Conformal geometry and quasiregular mappings, Lecture Notes in Math. V 1319, Springer-Verlag, 1988.

[38] R. Schoen, The Role of Harmonic Mappings in Rigidity and Deformation Problems in Collection:Complex Geometry (Osaka,1990), Lectures Notes in Pure and Applied Mathematics, Dekker, New York 143 (1993), 179-200. 
[39] H. Lang, A condition that a continuously deformed, simply connected body does not penetrate itself AGTM report, University of Kaiserslautern, Germany, No. 271, 2007 http://www.ltd.tf.uni-erlangen.de/Team/Lang/HLang_Publications.htm.

[40] W. Rudin, Function Theory in the Unit Ball of $C^{n}$, Springer-Verlag, Berlin Heidelberg New York, 1980.

[41] S. E. Warschawski: On the higher derivatives at the boundary in conformal mapping, Trans. Amer. Math. Soc. 38 No. 2 (1935) 310-340.

Faculty of mathematics, Univ. of Belgrade, Studentski Trg 16, Belgrade, Serbia

E-mail address: miodrag@matf.bg.ac.rs 